University of North Florida

UNIVERSITY of

NORTH FLORIDA.

UNF Digital Commons

1990

\title{
Adaptive Histogram Equalization, a Parallel Implementation
}

Charles W. Kurak Jr.

University of North Florida

Follow this and additional works at: https://digitalcommons.unf.edu/etd

Part of the Computer Sciences Commons

\section{Suggested Citation}

Kurak, Charles W. Jr., "Adaptive Histogram Equalization, a Parallel Implementation" (1990). UNF Graduate Theses and Dissertations. 260.

https://digitalcommons.unf.edu/etd/260

This Master's Thesis is brought to you for free and open access by the Student Scholarship at UNF Digital Commons. It has been accepted for inclusion in UNF Graduate Theses and Dissertations by an authorized administrator of UNF Digital Commons. For more information, please contact Digital Projects.

(C) 1990 All Rights Reserved

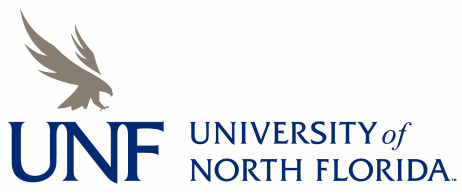




\section{ADAPTIVE HISTOGRAM EQUALIZATION}

A PARALLEL IMPLEMENTATION

by

Charles W. Kurak Jr.

A thesis submitted to the

College of Computer and Information Sciences

in partial fulfillment of the requirements for the degree of

Master of Science in Computer and Information Sciences

UNIVERSITY OF NORTH FLORIDA

COLLEGE OF COMPUTER AND INFORMATION SCIENCES

December, 1990 
The thesis "Adaptive Histogram Equalization: A Parallel Implementation" submitted by Charles W. Kurak Jr. in partial fulfillment of the requirements for the degree of Master of Science in Computer and Information Sciences has been

Approved by the thesis committee:

Signature Deleted

Thesis Adviser and Committee Chairman

Signature Deleted

Signature Deleted
Date
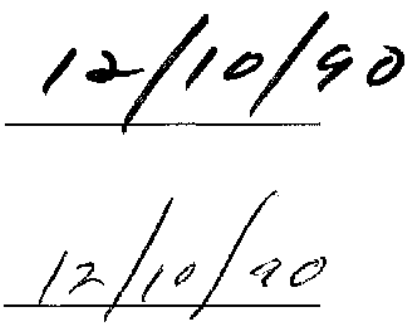

\section{$12 / 10 / 90$}

Accepted for the College of Computer and Information Sciences:

Signature Deleted

$$
12 / 10 / 90
$$

Interim Dean

Accepted for the University:

Signature Deleted

Vice-President for Academic Affairs 


\section{ACKNOWLEDGEMENTS}

I wish to thank my advisor and thesis committee chairman, Dr. Yap S. Chua, for his continuous support, advice, and encouragement during this work. The members of my committee also deserve credit for their support. Dr. Ralph M. Butler was extremely helpful with parallel algorithm design and by supplying the parallel programming package. Dr. Charles N. Winton, as always, continued to offer encouragement and material support throughout the project.

I wish to acknowledge the support I have received from members of the University of North Carolina at Chapel Hill community. Dr. Stephen M. Pizer introduced me to Adaptive Histogram Equalization and has been supportive of my efforts with his time and suggestions. Dr. James M. Coggins provided continual support during the project. Dr. Graham Gash aided with the 'usr/image' software developed at UNC-CH. This package was one of the tools utilized for data conversion and interactive image display. Mr. Robert Cromartie aided in the verification of the border mirroring methodology and the image results in general. The UNC Medical Image Display Research Group provided the medical images.

Saving the most important for last, my ever-supportive and cherished wife, Beverly, helped me through the long hours and seemingly endless nights and weekends. Her background as a businesswoman, an educator, and a loyal wife and mother continues to be an asset to my efforts. 


\section{CONTENTS}

List of Figures $\ldots \ldots \ldots \ldots \ldots \ldots \ldots \ldots \ldots \ldots \ldots$ vi

Abstract $\ldots \ldots \ldots \ldots \ldots \ldots \ldots \ldots \ldots \ldots \ldots \ldots \ldots$ vii

Chapter $1:$ Introduction $\ldots \ldots \ldots \ldots \ldots \ldots \ldots \ldots \ldots \ldots \ldots \ldots$

1.1 Digital Image Processing Historical Overview $\ldots \ldots \ldots \ldots \ldots$

1.2 Histogram Equalization $\ldots \ldots \ldots \ldots \ldots \ldots \ldots$

1.3 Adaptive Histogram Equalization (AHE) $\ldots \ldots \ldots \ldots \ldots$

1.4 AHE Limitations $\ldots \ldots \ldots \ldots \ldots \ldots \ldots \ldots$

1.4 .1 Time Performance $\ldots \ldots \ldots \ldots \ldots \ldots \ldots \ldots$

1.4.2 Edge Considerations $\ldots \ldots \ldots \ldots \ldots \ldots$

1.4.3 Excessive Computations $\ldots \ldots \ldots \ldots \ldots \ldots$

1.5 Parallel Processing $\ldots \ldots \ldots \ldots \ldots \ldots \ldots \ldots$

1.5.1 Parallel Programming Paradigms . . . . . . . . . . . 10

1.5.2 Message Passing Paradigm $\ldots \ldots \ldots \ldots \ldots \ldots \ldots$

1.5.3 Model Selection . . . . . . . . . . . . . . . . 12

Chapter 2: Hypothesis $\ldots \ldots \ldots \ldots \ldots \ldots \ldots \ldots \ldots \ldots \ldots$

Chapter 3: Implementation $\ldots \ldots \ldots \ldots \ldots \ldots \ldots \ldots \ldots$

$3.1 \mathrm{C}$ language and Unix Operating System . . . . . . . . . 14

3.2 Message Passing Paradigm $\ldots \ldots \ldots \ldots \ldots \ldots \ldots$

3.3 Multiple image copies $\ldots \ldots \ldots \ldots \ldots \ldots \ldots \ldots$

3.4 Image Border $\ldots \ldots \ldots \ldots \ldots \ldots \ldots \ldots \ldots \ldots$

3.5 Value Look-up Table . . . . . . . . . . . . . . 20

3.6 Coordination between Master and Slaves $\ldots \ldots \ldots \ldots 22$ 
Chapter 4: Tools

4.1 DISPLAY for the PC ................ 26

4.2 'disp' for the Sun workstation with $\mathrm{X}$ Windows $\ldots \ldots \ldots 27$

4.3 Image and Histogram Production for Postscript $\ldots \ldots$. . . . . . . 27

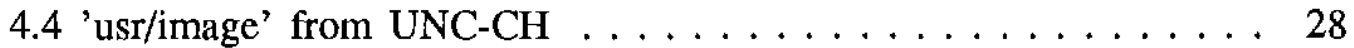

4.5 Thesis publication software $\ldots \ldots \ldots \ldots \ldots$

Chapter 5: Testing \& Results ... . . . . . . . . . . . . . . . . 29

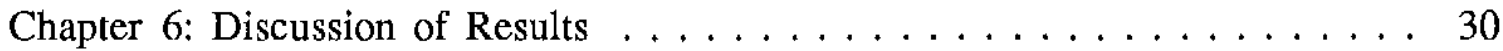

Chapter $7:$ Conclusion $\ldots \ldots \ldots \ldots$

Chapter 8: Future Work . . . . . . . . . . . . . . . . . . . . . . 39

8.1 SIMD architecture $\ldots \ldots \ldots \ldots \ldots \ldots$

8.2 Interactive viewing of image during processing . . . . . . . 40

8.3 The AHE Family of Algorithms . . . . . . . . . . . . 41

8.4 Three-dimensional AHE ................... 41

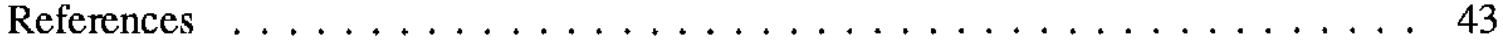

Appendix A: Source code for Parallel AHE (master process) $\ldots \ldots$. . . . . 45

Appendix B: Source code for Parallel AHE (slave process) $\ldots \ldots \ldots$. . . . . 54

Appendix C: Test Results . . . . . . . . . . . . . . . . . . 60

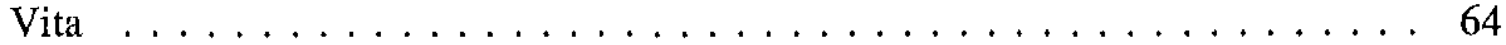




\section{FIGURES}

Figure $1:$ MRI Scan - Original Image $\ldots \ldots \ldots \ldots \ldots \ldots \ldots$

Figure 2: MRI Scan - after Contrast Stretching $\ldots \ldots \ldots \ldots \ldots$

Figure 3: Brain - Original Image $\ldots \ldots \ldots \ldots \ldots \ldots \ldots \ldots$

Figure 4: Brain - after Contrast Stretching $\ldots \ldots \ldots \ldots \ldots$

Figure 5: The AHE Algorithm $\ldots \ldots \ldots \ldots \ldots \ldots \ldots$

Figure 6: Number of required comparisons $\ldots \ldots \ldots \ldots \ldots \ldots \ldots$

Figure 7: One possible method of handling borders $\ldots \ldots \ldots \ldots \ldots$

Figure 8: Chest - after AHE with contextual region of $16 \times 16 \ldots \ldots 19$

Figure 9: Chest - after AHE with contextual region of $32 \times 32 \ldots \ldots 19$

Figure 10: Mirroring across the image boundaries $\ldots \ldots \ldots \ldots$

Figure 11: Message Passing Model $\ldots \ldots \ldots \ldots \ldots \ldots \ldots$

Figure 12: Master Process - Pseudo-Code . . . . . . . . . . . . 25

Figure 13: Slave Process - Pseudo-Code $\ldots \ldots \ldots \ldots \ldots \ldots \ldots$

Figure 14: Chest - Original Inage $\ldots \ldots \ldots \ldots \ldots \ldots \ldots \ldots \ldots$

Figure 15: Chest - after AHE with contextual region of $64 \times 64 \ldots \ldots 31$

Figure 16: Brain - Original Image $\ldots \ldots \ldots \ldots \ldots \ldots \ldots \ldots \ldots$

Figure 17: Brain - after AHE with contextual region of $64 \times 64 \ldots 32$

Figure 18: AHE Results - Contextual Region of $8 \times 8 \ldots \ldots 33$

Figure 19: AHE Results - Contextual Region of $16 \times 16 \ldots \ldots 33$

Figure 20: AHE Results - Contextual Region of $32 \times 32 \ldots \ldots \ldots$

Figure 21: AHE Results - Contextual Region of $64 \times 64 \ldots \ldots \ldots 34$

Figure 22: AHE Results - 26-node machine $\ldots \ldots \ldots \ldots \ldots \ldots$

Figure 23: Dividing the image into interlaced sub-problems $\ldots \ldots \ldots \ldots 41$ 


\section{ABSTRACT}

Adaptive Histogram Equalization (AHE) has been recognized as a valid method of contrast enhancement. The main advantage of AHE is that it can provide better contrast in local areas than that achievable utilizing traditional histogram equalization methods. Whereas traditional methods consider the entire image, AHE utilizes a local contextual region.

However, AHE is computationally expensive, and therefore time-consuming. In this work two areas of computer science, image processing and parallel processing, are combined to produce an efficient algorithm. In particular, the AHE algorithm is implemented with a Multiple-Instruction-Multiple-Data (MIMD) parallel architecture. It is proposed that, as MIMD machines become more powerful and prevalent, this methodology can be applied to not only this particular algorithm, but also to many others in its class. 


\section{Chapter 1}

\section{INTRODUCTION}

Digital image processing has aided in the enhancement and restoration of images for over a quarter of a century. This work focuses on one particular algorithm among the many available. In particular, it will be proposed that the algorithm in question can be improved with the application of knowledge from a separate branch of computer science. To enable the reader to better understand the algorithm, relevant background information will be presented.

\subsection{Digital Image Processing Historical Overview}

Digital image processing techniques had their significant inception in the 1960s with the space program. The requirement to enhance the quality of the photographs returned by the early space probes motivated research in this area. Originally this technology was applied to space imagery, however it was soon realized that other areas could also benefit. Consequently, the needs of the medical field attracted the attention of researchers. The methods utilized by the space industry were applied. With the advent of the X-ray and later Nuclear Magnetic Resonance Imaging (NMRI or MRI), Positron Emission Tomography (PET Scans), Computerized Assisted Tomography (CAT Scans), and Ultra-sound Imaging, the quantity of medical data increased dramatically. Simultaneously researchers sought better methods to enhance these images. 
The test data utilized for this work originated from three different types of data collection methods. The chest image is one slice of a CAT scan (see Figure 14 on page 31). The left facing head is slice number 50 of a 109-slice MRI scan (see Figure 1 on page 4). The right facing head labeled 'brain' is a portal image taken during Radiotherapy Treatment (see Figure 16 on page 32). Each file arrives for enhancement in a raw image format. In raw image format the intensity values are contained in a file in row major order, with multiple planes being stored consecutively from front to back.

\subsection{Histogram Equalization}

One of the most basic and simple, yet powerful tools in image enhancement is the histogram. This tool is simply a frequency count of the intensity levels of each digitized point, or pixel, contained in the image. Utilizing the information contained in a histogram allows us to improve the contrast of an image. This information may be hidden from the human eye; however it is readily acquired by use of a computer. Whereas the Human Visual System (HVS) can only distinguish approximately 100 levels of gray shades, the computer can detect an almost infinite number of levels. The practical limiting factor for the computer is the number of various intensity levels recognizable by the digitizing equipment.

For example, using the histogram, under-developed or over-developed photographs can be restored or enhanced to produce an image usable by the HVS. Assuming the histogram reveals a number of intensity levels all located in the low intensity range, each current value can be mapped to a new level so that the new histogram 
is scaled to cover the entire range of available intensity levels. Note the images and their accompanying histograms in Figures 1 and 2 on page 4 .

\subsection{Adaptive Histogram Equalization}

The Histogram Equalization method of contrast enhancement functions extremely well for images that are underexposed or overexposed, i.e. images with very little overall contrast. However, there exist images whose histograms cover the entire spectrum of intensity values but reveal little contrast in localized areas (see Figures 3 and 4 on page 5). In this case a variation of histogram equalization can be applied. Developed independently by Hummel [Hummel75, Hummel77], Ketcham [Ketcham76], and Pizer [Pizer81a, Pizer81b], Adaptive Histogram Equalization (AHE) has been successfully applied to images obtained from numerous sources. Although this thesis concentrates on medical imagery, the success of the method used depends on the characteristics of the image, not the content. The identical methodology can be applied to other areas of interest.

The technique is quite simple. The algorithm is included in Figure 5 on page 6. Each pixel is ranked by its intensity level as compared to its neighboring pixels' intensity values. The pixel is then assigned a new value in the available intensity range proportionate to its rank. For example, if a pixel's rank is \#8 of 64 and the available intensity range of the display device is $0-255$, its new value would be oneeighth of 255 or 32 . This new value is assigned to a second image (an output image) so as to not disturb the original ranking of each of the pixels. 
DISPLAY - Image Processing Sottware by Charles Kurak - Sep 231990 - University of North Fborida

MRI Scan - Original Image.
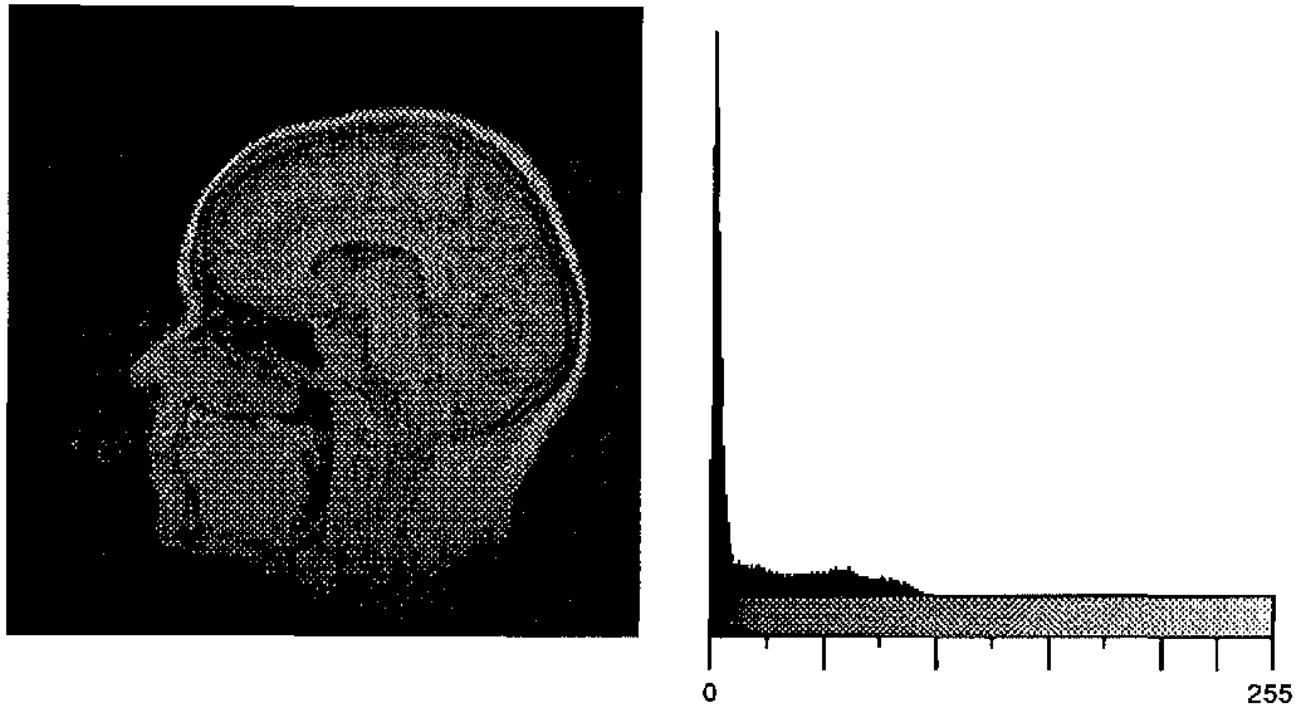

Figure 1: MRI Scan - Original Image.

DISPLAY - Image Processing Sottware by Charles Kurak - Sep 231990 - University of North Florida

MRI Scan - after Contrast Stretching.
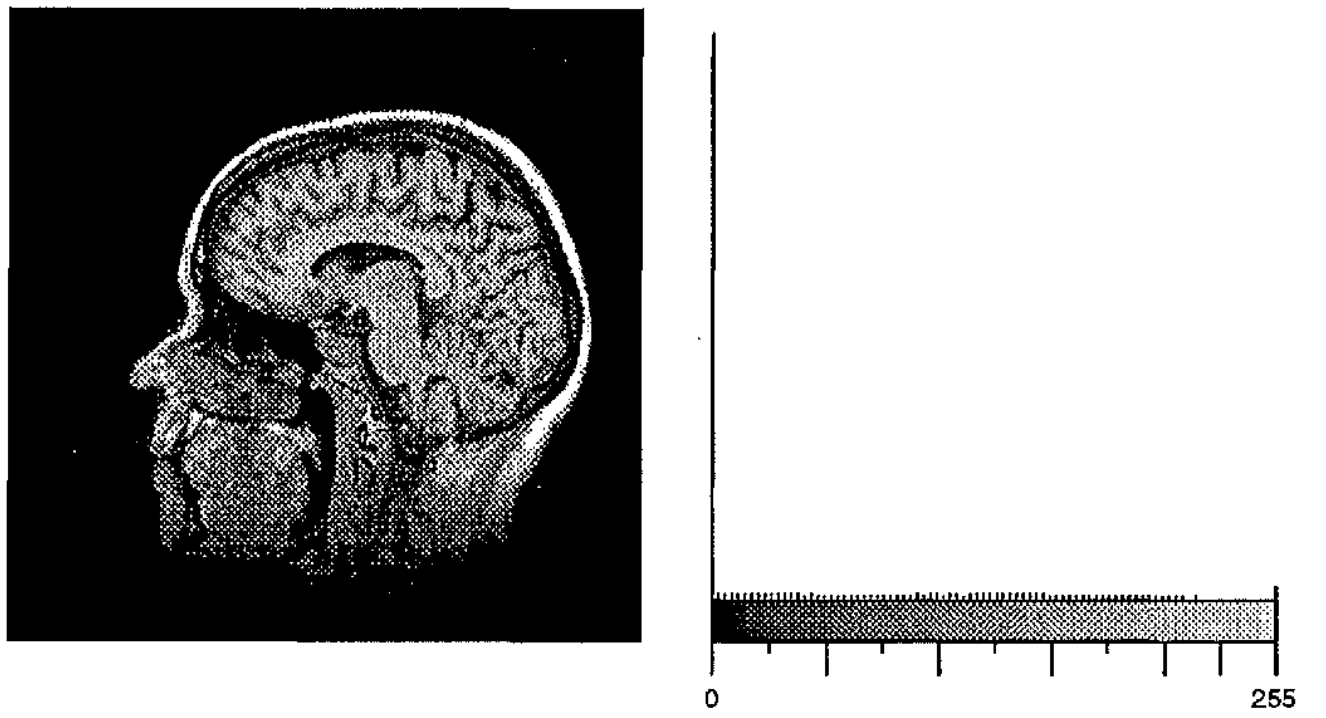

Figure 2: MRI Scan - after Contrast Stretching. 
DISPLAY - Image Processing Sottware by Charles Kurak - Sep 231990 - University of North Florida

Brain: original image.
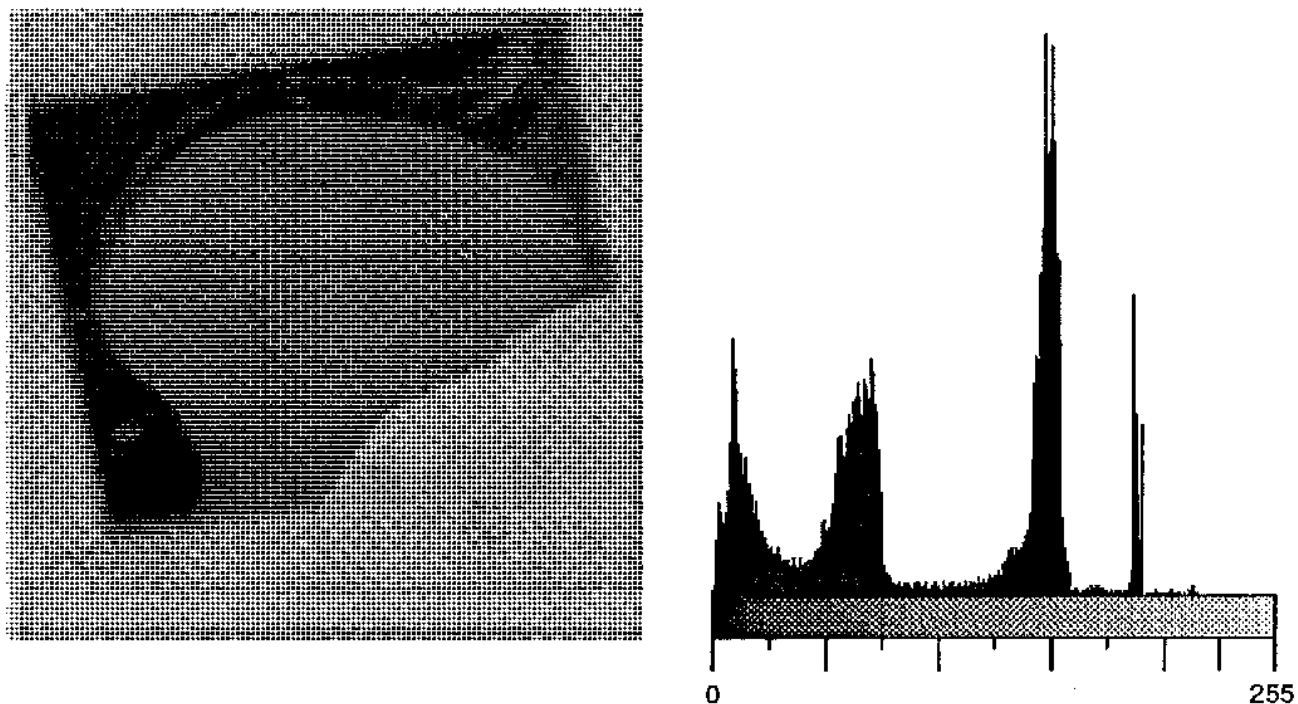

Figure 3: Brain - Original Image.

DISPLAY - Image Processing Soltware by Charles Kurak - Sep 231990 - University of North Florida

Brain: after contrast stretching.
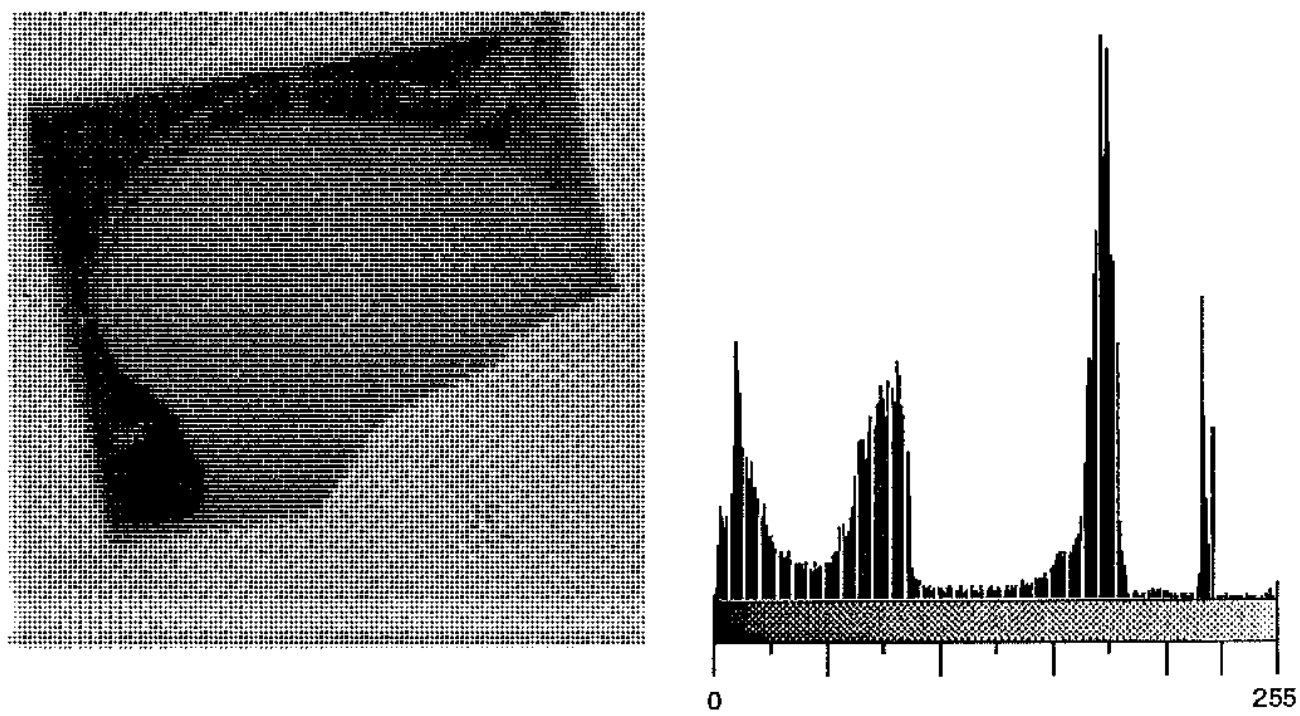

Figure 4: Brain - after Contrast Stretching. 


\section{$\underline{\text { AHE Algorithm }}$}

for each $(x, y)$ in image do

l

rank $=0$

for each $(i, j)$ in contextual region of $(x, y)$ do

l

if image $[x, y]>$ image $[i, j]$ then

$$
\text { rank }=\text { rank }+1
$$

\}

output $[\mathrm{x}, \mathrm{y}]=\operatorname{rank} * \max$ intensity $/(\#$ of pixels in contextual region)

\}

Figure 5: The AHE Algorithm.

This method enhances the contrast based on the local area or contextual region rather than the entire image. AHE is currently being utilized in both research at the University of North Carolina at Chapel Hill, and in production systems such as Mayo Clinic's "Analyze" software.

\subsection{AHE Limitations}

While Adaptive Histogram has the advantage of being able to enhance contrast in local areas, there is a price to pay. Three areas of concern surfaced during the initial investigation: time, border considerations, and excessive computations. These are discussed below. 


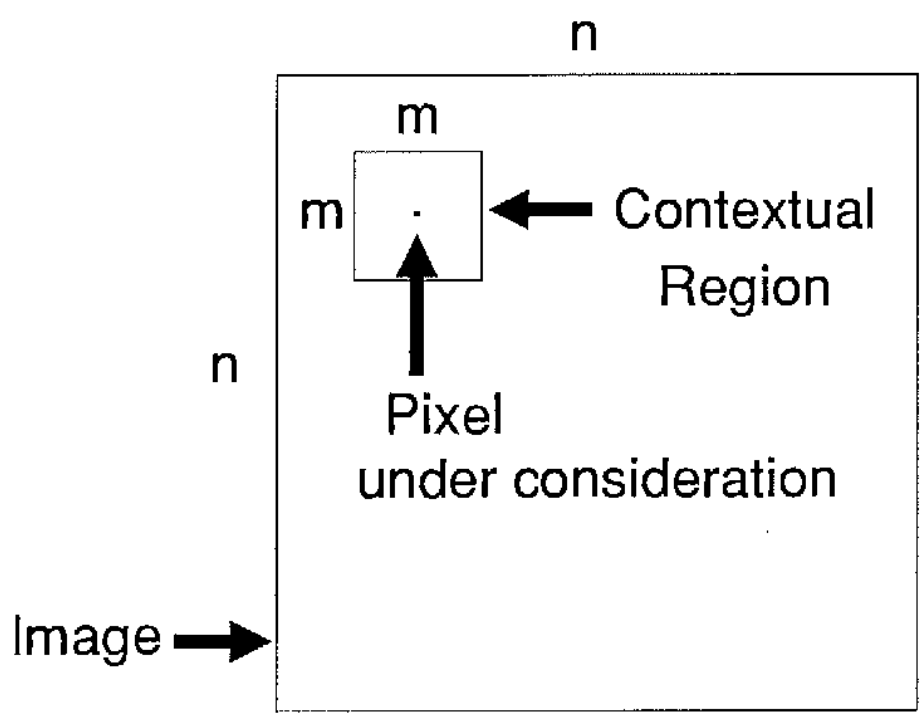

Number of comparisons required for AHE is $m^{2} \times n^{2}$, where $m$ is the size of the contextual region and $\mathrm{n}$ is the size of the image.

Figure 6: Number of required comparisons.

\subsubsection{Time Performance}

Unfortunately, applying AHE to an image is computationally expensive. Each image requires $\mathrm{m}^{2} \mathrm{x} \mathrm{n}^{2}$ comparisons where $\mathrm{m}$ is the size of the contextual region and $\mathrm{n}$ is the size of the image. (See Figure 6 above.) For this to be a truly valid tool for a radiologist or a medical doctor, the processing must be completed in a reasonable amount of time. Initial studies by the author determined that in excess of one hour on a 4 MIPS Sequent Symmetry machine was required to process a $512 \times 5128$-bit image with a $64 \times 64$ contextual region. Consultation with Dr. Pizer and Mr. Robert Cromartie at UNC-CH indicated that lowering the processing times would be extremely beneficial. 
Three approaches were considered. First, simply wait until faster hardware is available. Second, one could build specialized hardware. MAHEM [Austin87] is one such research effort that utilizes a parallel processing architecture. Third, search for an alternative. In this work the author proposes one alternative: parallelism on commercially available hardware. The majority of the remainder of this work addresses this approach.

\section{1,4.2 Border Considerations}

An issue that surfaced during the investigation of the AHE algorithm is that of handling pixels whose contextual regions overlapped the borders. Current literature [Ericksen90] suggests that for convenience, the contextual region indices simply utilize a wrap-around technique. As suggested by the author to Dr. Pizer during a personal meeting in May 1990, this technique is invalid. For example, if the area near the border under consideration is relatively dark (low intensity values are present), and the values on the opposite edge are relatively light (high intensity values are present), the resulting rank calculation would be unsatisfactorily biased.

A mirroring technique has been suggested by the author. This technique has been implemented in this work and is explained in Section 3.4.

\subsubsection{Excessive Computations}

In addition to the number of comparisons required by the AHE algorithm, an additional issue surfaced during the implementation. This is the number of 
excessive computations required to calculate each new intensity value. In the AHE algorithm in Figure 5, on page 6, note the calculation for the new intensity value, output $[x, y]$. This requires one multiplication operation and one division operation for each pixel. For example, in a $256 \times 256$ image there would be $65,536\left(256^{2}\right)$ integer multiplications and 65,536 $\left(256^{2}\right)$ integer divisions required.

One method of reducing the number of calculations is to precalculate the max_intensity/(\# of pixels in contextual region). This eliminates an integer division calculation, however it replaces the integer multiplication with a floating point multiplication, which may be more time consuming.

Another method, which reduces the total number of calculations to the number of pixels in the contextual region, was devised utilizing a look-up table. The method is discussed in Section 3.5 .

\subsection{Parallel Processing}

The concept of accomplishing more work in less time by employing additional resources is not original by any consideration. After studying the AHE algorithm it can be determined that one can take advantage of the positive aspects of parallel processing. The following is a brief discussion of the programming models available, the one selected for the implementation, and reasons for this choice. 


\subsubsection{Parallel Processing Programming Paradigms}

There are three well known programming models for parallel processing in an MIMD environment. These are the Askfor-Monitor, Message Passing, and Communicating Clusters [Boyle87]. The Askfor-Monitor requires a shared memory machine. That is, each processor must have access to a common memory area that is shared by all of the processors. The processes communicate through this common area. The monitor is a data structure that controls the activities of the processes.

Message Passing can operate either across a network or on a shared memory machine. The processes communicate by passing messages. One process is designated as the master and controls the activities of the other processes, also known as slaves. The ability to operate across a network allows the use of nonmulti-processing hardware to function together in a multi-processing fashion.

The third model is Communicating Clusters. Essentially this is a combination of Askfor Monitors and Message Passing. A master process on one machine invokes processes on other machines that have shared memories. One process on each of these machines is designated by the master to be a cluster master. They in turn designate the other processes on their machine to be local slaves. Each cluster coordinates its work via an Askfor-Monitor in shared memory. The master coordinates the work among the clusters via Message Passing. Thus, the Communicating Clusters model can be custom-built depending on the available hardware. 


\subsubsection{Message Passing Paradigm}

The model selected for this work was Message Passing. This decision was based on portability across several different architectures. A brief example is presented here to acquaint the reader with the Message Passing model.

Suppose we were given the task of finding all of the prime numbers within a given range. With a large enough range this becomes non-trivial. Also, suppose we were given multiple processors whose processes could communicate via messages. We could then take advantage of a parallel algorithm for solving the problem.

One process is designated as the initiating process (master). Its function is to read the problem, decompose the problem into sub-problems, activate processes on other processors (slaves), hand out the work, collect the answers, and report a solution. The remaining processes perform the actual work, under the control of the master.

The master is the first to be invoked. It in turn invokes the proper number of slave processes as indicated in a user-defined parameter setup file. The master then divides the work into a series of sub-problems and places a representation for each in a problem queue for dispatching to the slaves. As each slave is initiated, it sends a message to the master requesting work. The master places a representation for each slave in a slave queue indicating that it is available to perform work. The master then proceeds to the main control loop of the program. If the problem queue is not empty, the master in turn dispatches the sub-problems to the 
appropriate slaves. Each slave computes its sub-problem, sends its solution back to the master, and requests additional work. When the problem queue is empty and the slave queue contains all of the invoked slaves, the problem is declared complete, and the master leaves the main control loop. The master sends a control message to each slave to terminate. The complete solution is reported to the user, normally as an output file. The master then terminates.

\subsubsection{Model Selection}

The Message Passing model was selected for this work due to its ability to operate both in a shared memory environment and across a network. The parallel programming package utilized for the implementation was designed for a shared memory machine. However, there is a compatible package that can be substituted without any requirement for source code modification. The desire to utilize this code as an ongoing research tool influenced the selection of the most portable model. 


\section{Chapter 2}

\section{HYPOTHESIS}

There are parallel processing techniques which will substantially improve the time performance of Adaptive Histogram Equalization.

Given the large amount of similar computation necessary to complete the AHE algorithm, the premise is that applying parallel processing techniques will greatly reduce the amount of overall time required to process an image. Assuming that one image requires amount $\mathrm{k}$ time, then dividing the work among $\mathrm{n}$ processors would yield an ideal completion time of $\mathrm{k} / \mathrm{n}$. Another way of saying this, is that one could obtain a speed-up of factor $n$.

The ideal speed-up of factor $\mathrm{n}$ is not realistic. There is necessary overhead relating to the coordination of the $\mathrm{n}$ processes, problem distribution, and solution reporting. The remaining question is then, how closely can one approach the ideal? 
Chapter 3

\section{IMPLEMENTATION}

\section{1 'C' Language and Unix Operating System}

The programming language ' $\mathrm{C}$ ' was selected for this implementation. Other languages could have been utilized. However the parallel programming package supplied by Dr. Ralph Butler [Butler90] is implemented in the language ' $\mathrm{C}$ '. For reasons of ease and compatibility the choice was obvious. Also, future work interfacing this software with the ' $\mathrm{X}$ Windows' software system will need to be in 'C'. The Unix operating system was selected, as Dr. Butler's package is implemented to run under Unix.

\subsection{Message Passing Paradigm}

This particular model was selected from [Boyle87] mainly due to its portability. As mentioned in 1.5.1, Message Passing was selected for its portability across a variety of platforms. The source code for this work is included in Appendices A \& B. The majority of the code is related to the coordination of the work between the master and the slaves.

The master's responsibilities are to determine the problem and number of slaves to be used, invoke the slaves, coordinate the work, gather the solution, terminate the 
slaves' existence, and output the solution. The master source code is included in pmaster.c in Appendix A.

Each slave's responsibility is to load the image into its local memory, compute sections of the image as requested by the master, and pass the completed problem solutions to the master. The slave source code is included in pslave.c in Appendix B.

\subsection{Multiple Image Copies}

During the design phase it was determined that passing a problem from the master to a slave can be handled in three different methods. First the master can send a copy of the sub-image to be processed to a slave. Given a sub-problem size of 64 $\mathrm{x}$ 64, this would be 4096 bytes. Also, given that the algorithm requires the values of pixels in neighboring areas, and given a typical $64 \times 64$ contextual region size, the overall sub-image size that needs to be transmitted is $128 \times 128$ or 16,384 bytes. This would have to be sent for each sub-problem. Given a $256 \times 256$ image, and a sub-problem size of $64 \times 64$, this would yield 16 sub-problems. Thus, a total of 262,144 bytes $(16,384 \times 16)$ would need to be transmitted.

A second method would be to send the entire image at once. Then only a representation of each sub-problem would need to be transmitted for each piece of work. This would involve transmitting 65,536 bytes. This is still a large amount of overhead. 
A third method, where only the file name be sent once, then a representation of each sub-image need be transmitted, can be handled with 8 bytes for each subproblem. The original image file would be located on a network file server. Two byte integers for each of the following are required: the horizontal and vertical positions of the upper left corner of the sub-problem in the original image, the problem size (given a square problem), and the contextual region size (a contextual region is by definition square). If a requirement is later added for non-square problems, one additional integer would be required. Given the same 16 subproblems as above, this would result in a transmission of only 128 bytes $(16 \times 8)$.

In this work, the third method was utilized. This resulted in the savings of 262,106 transmitted bytes over the first method, and a savings of 65,536 bytes over the second method. The trade-off is that each slave must load a copy of the original image from secondary storage.

\subsection{Image Border}

As mentioned in 1.4.2, current literature suggestions for handling the border are not valid. It is the opinion of the author that a user of a processed image would expect that the AHE algorithm technique should perform equally well in all areas of the image. It is widely known that most measuring devices are more accurate in their central regions than either end of their scales. However, that should not preclude every attempt to provide as much accuracy as possible throughout. The obvious solution for handling the inaccuracies of the image edges is to collect more data. This, besides being infeasible given existing images and the limitations of the data 


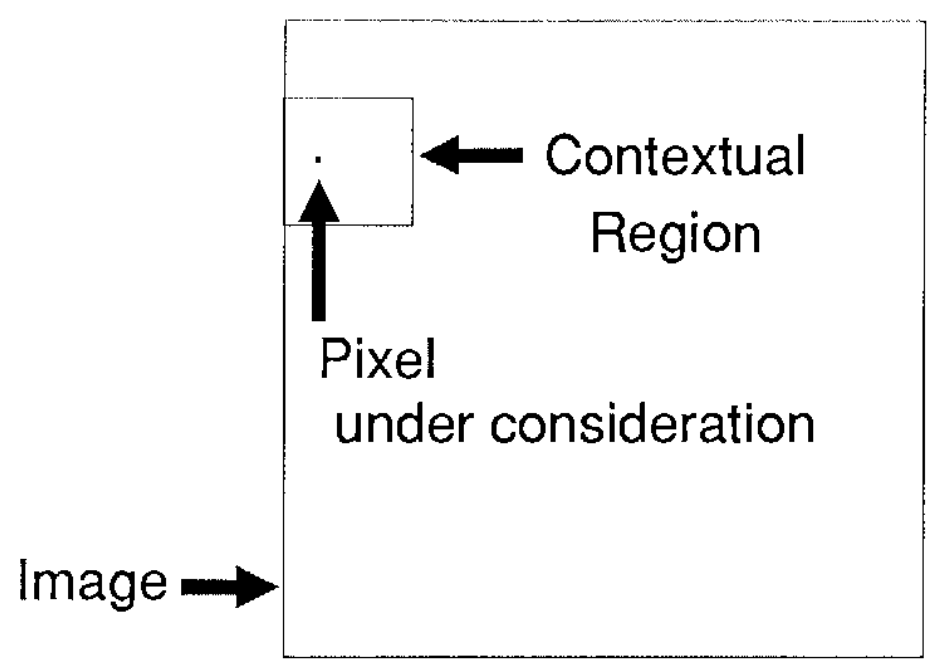

One possible method of handling borders:

Hold the contextual region in place as the point under consideration approaches the image edge.

Figure 7: One possible method of handling borders.

collection devices, only delays the problem. There would then exist new edges with which to contend. The user would then expect accuracy near these edges as well.

One possible technique would be to hold the contextual region in place at the edge as the point under consideration approaches the edge (see Figure 7 above). This adds special cases to the algorithm. As the contextual region overlaps the edge, it must be 'backed up' to remain on the image. This must be considered in both the horizontal and vertical directions. There are special cases of left, right, top, bottom, upper-left, upper-right, lower-left, and lower-right. This introduces additional comparisons and referencing requirements into the algorithm implementation. Each additional computation requires time. Time is a precious commodity not freely available here. 
Also, as the point of consideration moves off the center of the contextual region, values that are now more than half a width of a region away are being utilized in its computation. This also yields a bias.

Another technique would be to reduce the size of the contextual region. However as the size of the contextual region diminishes, the number of possible outcomes decreases. This results in an over-enhanced image near the edges. This would not be compatible with the remainder of the image. An example of varying the contextual region size is depicted in Figures 8 and 9 on page 19. This method also results in excessive comparisons and computations due to the necessity of detecting and handling special cases, thus resulting in a reduction of time performance.

The author has developed a method for mirroring the image across the border. Several benefits are derived from this technique. First, it eliminates special cases during the execution of the algorithm. An oversized image structure is built in primary storage with the original image placed in the center. The remainder of the structure is 'filled in' by mirroring the values across the image boundary. (See Figure 10 on page 20.) This eliminates numerous calculations. Second, each new pixel's value is computed by considering only values in its contextual region. That is, no compared pixel is more than half a region away in either the horizontal or vertical direction. Third, this eliminates the bias that occurs with a wrap-around technique. 


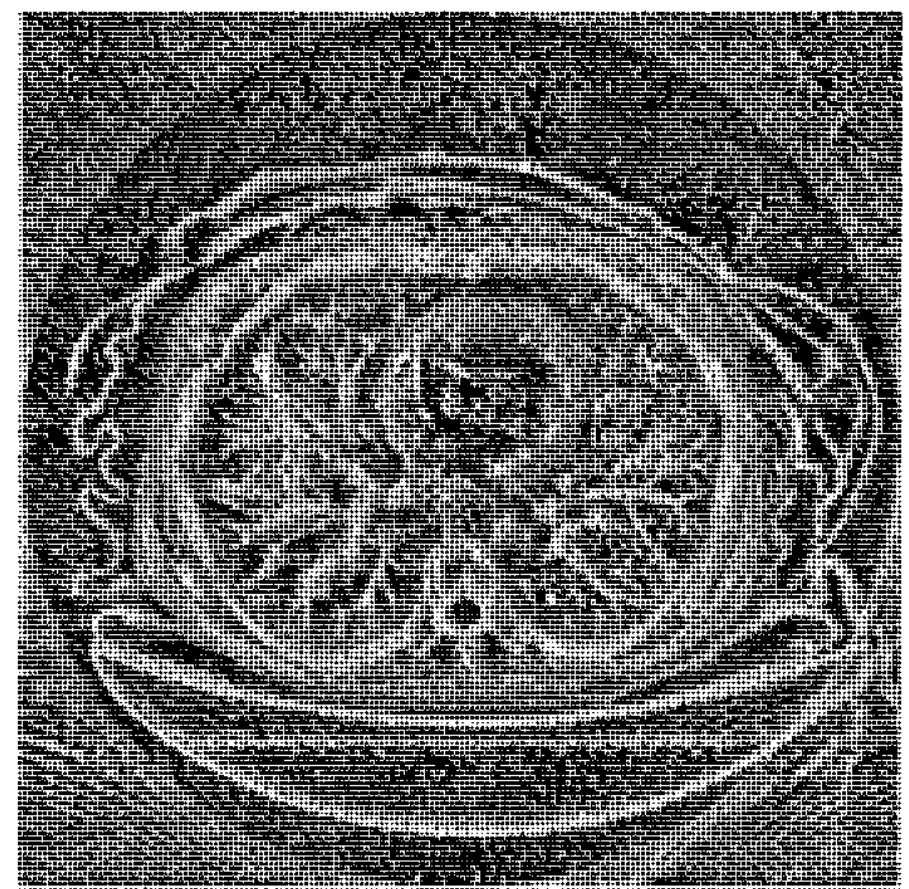

Figure 8: Chest - after AHE with contextual region of $16 \times 16$.

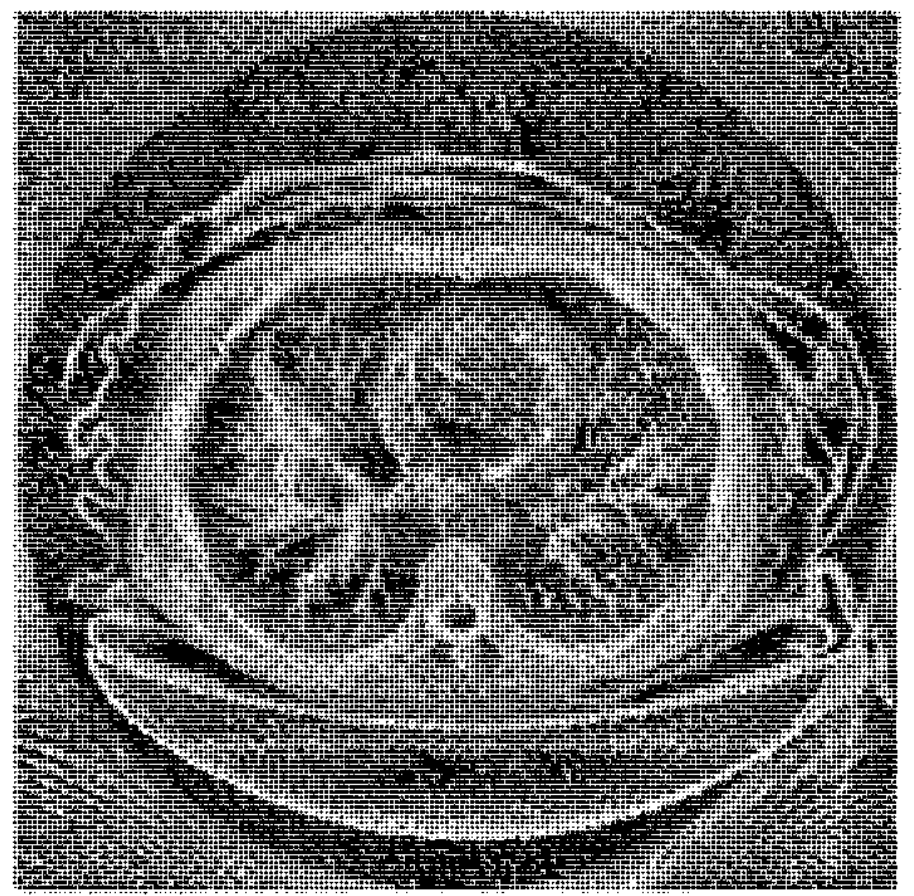

Figure 9: Chest - after AHE with contextual region of $32 \times 32$. 
Original Image is located in center square.

Array in primary storage includes mirrored border values.

\begin{tabular}{|rrrrrrrrrrrrrrrrr}
\hline 1 & 27 & 26 & 25 & 25 & 26 & 27 & 28 & 29 & 30 & 31 & 32 & 32 & 31 & 30 & 8 \\
20 & 1 & 18 & 17 & 17 & 18 & 19 & 20 & 21 & 22 & 23 & 24 & 24 & 23 & 8 & 21 \\
12 & 11 & 1 & 9 & 9 & 10 & 11 & 12 & 13 & 14 & 15 & 16 & 16 & 8 & 14 & 13 \\
4 & 3 & 2 & 1 & 1 & 2 & 3 & 4 & 5 & 6 & 7 & 8 & 8 & 7 & 6 & 5 \\
4 & 3 & 2 & 1 & 1 & 2 & 3 & 4 & 5 & 6 & 7 & 8 & 8 & 7 & 6 & 5 \\
12 & 11 & 10 & 9 & 9 & 10 & 11 & 12 & 13 & 14 & 15 & 16 & 16 & 15 & 14 & 13 \\
20 & 19 & 18 & 17 & 17 & 18 & 19 & 20 & 21 & 22 & 23 & 24 & 24 & 23 & 22 & 21 \\
28 & 27 & 26 & 25 & 25 & 26 & 27 & 28 & 29 & 30 & 31 & 32 & 32 & 31 & 30 & 29 \\
36 & 35 & 34 & 33 & 33 & 34 & 35 & 36 & 37 & 38 & 39 & 40 & 40 & 39 & 38 & 37 \\
44 & 43 & 42 & 41 & 41 & 42 & 43 & 44 & 45 & 46 & 47 & 48 & 48 & 47 & 46 & 45 \\
52 & 51 & 50 & 49 & 49 & 50 & 51 & 52 & 53 & 54 & 55 & 56 & 56 & 55 & 54 & 53 \\
60 & 59 & 58 & 57 & 57 & 58 & 59 & 60 & 61 & 62 & 63 & 64 & 64 & 63 & 62 & 61 \\
60 & 59 & 58 & 57 & 57 & 58 & 59 & 60 & 61 & 62 & 63 & 64 & 64 & 63 & 62 & 61 \\
52 & 51 & 57 & 49 & 49 & 50 & 51 & 52 & 53 & 54 & 55 & 56 & 56 & 64 & 54 & 53 \\
44 & 57 & 42 & 41 & 41 & 42 & 43 & 44 & 45 & 46 & 47 & 48 & 48 & 47 & 64 & 45 \\
57 & 35 & 34 & 33 & 33 & 34 & 35 & 36 & 37 & 38 & 39 & 40 & 40 & 39 & 38 & 64 \\
\hline
\end{tabular}

Figure 10: Mirroring across the image boundaries.

3.5 Value Look-up Table (LUT)

As mentioned in Section 1.4.3, an issue that surfaced during the implementation of this work is the excessive number of computations in determining the new intensity values. Given an $\mathrm{m} \times \mathrm{m}$ contextual region, there are only $\mathrm{m}^{2}$ possibilities for each new pixel intensity. Given an $\mathrm{n} \times \mathrm{n}$ image, this requires $\mathrm{n}^{2}$ calculations to determine all of the new values. Since $\mathrm{n}^{2}$ is much greater than $\mathrm{m}^{2}$, many of the calculations are repetitive. A Look-up Table has been employed to avoid the 
redundant calculations. Since the new intensity values are required by each slave process, each slave builds its own LUT.

Another possibility was considered that would eliminate the need for a Look-up Table and the excessive multiplications and divisions. However, there needs to be some restrictions on the problem parameters. If we require the size of the contextual region to be a power of 2 , and the number of intensities available on the display device also to be a power of 2 , we can then reduce the calculations to register shifting. This may in fact be more expedient than a table look-up, however, for the purpose of the thesis and for general applicability, this method was not pursued.

This implementation under discussion takes a more general approach and does not limit the parameters to a specific set of values. The rationale behind this decision is that the size of the contextual region should be left to the user. Since the user needs to vary the region size to produce the best results as necessary, it would be overly restrictive to limit the available region sizes.

Whereas this discussion of excessive computations is not a feature of the parallel implementation, it is sound computing practice to streamline the sequential section of the code where possible. Thus it is mentioned here. 


\subsection{Coordination between Master and Slaves}

The Message Passing Paradigm was outlined in Section 1.5.2. The following description depicts the actions of the master and slaves as applied to the AHE algorithm. A graphic representation is included in Figure 11 on page 23. The accompanying pseudo-code is included in Figures 12 and 13 on page 25.

The master is the first process to be invoked. It determines the number of slaves to be utilized by reading a file in secondary storage. This is done in accordance with the parallel processing program package [BUTLER90]. The size of the contextual region, the size of the problem pieces, and the names of the input and output files are read from the command line.

As the master invokes each slave, it establishes the lines of communication between itself and each slave. The master also sends the name of the input file to each slave process. The slaves perform their start-up work, i.e. loading the image and building the mirrored borders. The master by this time has divided the original problem into sub-problems as determined by the input parameters. A representation of each problem is placed in a linked list structure, referred to as the problem queue. The master then enters its main control loop. Each slave, upon completion of its initialization tasks, transmits a request for work to the master.

During the main control loop, the master checks to see if the condition exists that the slave queue is non-empty and the problem queue is non-empty. If these conditions are met, the master then dispatches a sub-problem to a slave represented 


\section{Message Passing Model}

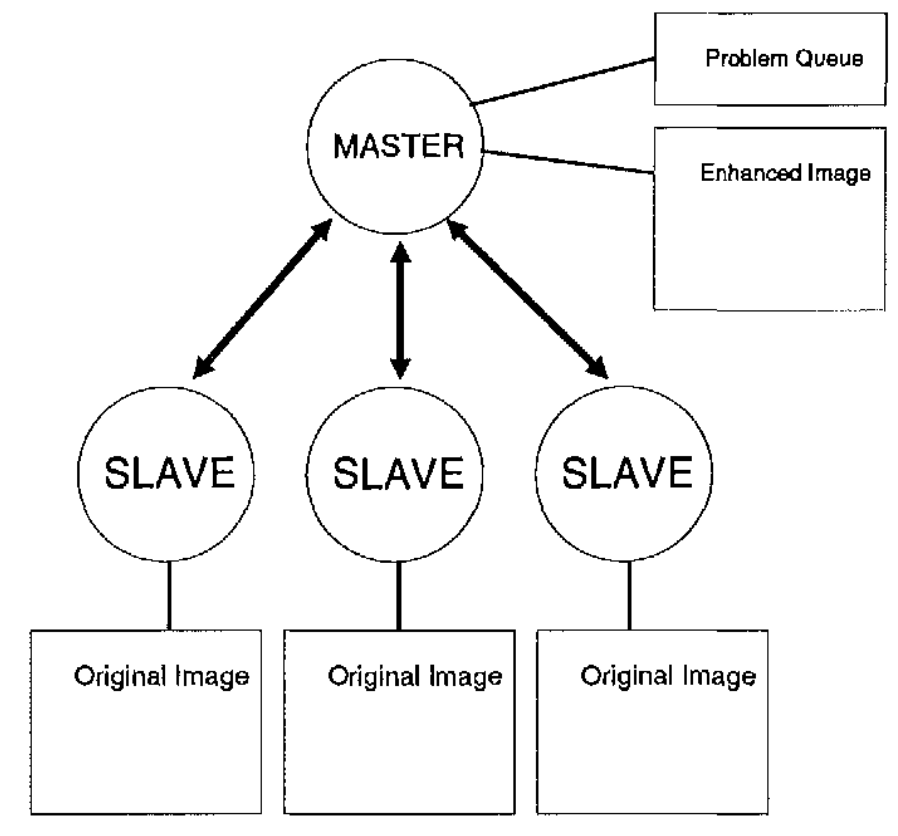

Figure 11: Message Passing Model.

on the slave queue. The appropriate piece of work and the slave are removed from their respective queues. As the master receives each request for work, it places a representation of the calling slave on a linked list slave queue.

The slave then receives the work, processes the sub-problem, and returns the resulting sub-image to the master. The master, upon receiving a message, determines the type of message, stores a copy of the sub-image in primary storage, then awaits the next message. This pattern continues until the master determines that all of the slaves are represented in the slave queue and the problem queue is empty. The problem has been completed at this point. 
The master completes its functions by sending the completed image to secondary storage, issuing a command for the slaves to terminate, waiting for the slaves to terminate, and outputting any statistics gathered for testing purposes. 


\section{Master Process Pseudo-code}

Create slaves

Read in parameters

Transmit control messages to slaves including image filename

Initialize solution data structure

Initialize slave queue

Initialize problem queue

Divide job into problems and place in problem queue

done $=$ FALSE

While (NOT done)

\{

While (slave queue is NOT empty) and (problem queue is NOT empty) Send a problem to a slave

Receive any message

If message type is REQUEST_.WORK then

Add slave to slave queue

If message type is SOLUTION then

Update solution data structure

If (ALL slaves are in slave queue) AND (problem queue is empty) then done $=$ TRUE

)

Output solution to secondary storage

Send ENDSIGNAL message to slaves to terminate

Wait for all slaves to terminate

Figure 12: Master Process - Pseudo-Code.

\section{$\underline{\text { Slave Process Pseudo-code }}$}

Receive message including image filename

Read image from secondary storage

Build mirrored borders

Send REQUEST_WORK message to master

while (received message type is NOT ENDSIGNAL)

(

Receive a message

If message type is DATA then

Perform AHE on sub-problem described in message

else

Send ENDSIGNAL to next slave in loop

J

Figure 13: Slave Process - Pseudo-Code. 
Chapter 4

\section{TOOLS}

Numerous software tools were utilized during the course of this work. Each of these were required to support the overall project. Their uses included presentation production, data conversion, and image viewing. These were in addition to the tools one would normally expect such as compilers, linkers, editors, etc. Several of these tools were developed by the author. These software endeavors were in addition to the main project implementation. Their description is included here to illustrate some of the additional research and development required by the author to complete this endeavor.

\subsection{DISPLAY for the PC}

One of the early research tools developed by the author was an interactive image processing program, DISPLAY. This was developed under MS-DOS for an IBMPC clone with EGA graphics capabilities. Its original development was in Pascal. Later the software was converted to C. DISPLAY will load a $256 \times 256 \times 8$ image from secondary storage into main memory. The image is then displayed in 16 color pseudo-color along with a histogram of the image.

DISPLAY is a menu-driven system that allows the user to perform point and area functions on the image. It will also allow several different output formats. The point operations include contrast enhancement, histogram stretching, and color 
inversion. The area operations include neighborhood averaging ( 8 or 9 elements), neighborhood median ( $3 \times 3$ or $5 \times 5$ ), edge detection, Adaptive Histogram Equalization with choice of contextual region size, and several other filters. The output functions include formatting the currently displayed image as a raw image file (256 x $256 \times 8$ ), or in Postscript format, with or without accompanying histogram.

The DISPLAY software has been extremely beneficial in producing both hardcopy images as well as overhead slides for several presentations during the course of this work. The majority of the images included in this presentation were produced by DISPLAY.

\section{2 'disp' for the Sun workstation with $\mathrm{X}$ Windows}

During the project it was necessary to view the images being processed without the expense and time required to produce a hard copy. The author developed 'disp', a display program built in ' $\mathrm{C}$ ' on the Sun workstation utilizing $\mathrm{X}$ Windows. It provided quick feedback during the development of the parallel AHE software. It was also beneficial to view the output images as the input parameters were varied.

\subsection{Image and Histogram Production for Postscript}

Some of the functions included in DISPLAY (See Section 4.1) were ported to the Unix environment. These included functions to produce Postscript files of images for presentation usage. These Postscript files were then 'viewed' either with 
Ghostscript 1.3, the NeXT's Preview facility, or sent to a Postscript capable printer for hardcopy production. As Postscript is a language unto itself, it was necessary for the author to learn a subset of the language to facilitate Postscript file generation.

\section{4 'usr/image' from UNC-CH}

The medical images included as test data for this work were supplied by the UNC Medical Image Display Research Group. This research organization is located at the University of North Carolina at Chapel Hill. The format of some of the images is unique to UNC-CH. The data was converted to raw image files with the utilization of a suite of software tools known to UNC-CH as 'usr/image' running under $\mathrm{X}$ Windows. This software proved quite worthy in converting images between formats so as to enable the transfer of images between the author and members of the UNC-CH community.

\subsection{Thesis publication software}

In addition to the image production software mentioned above, the balance of this publication was produced with Harvard Graphics, Quattro-Pro, and Wordperfect. 


\section{Chapter 5}

\section{TESTING AND RESULTS}

Program testing was conducted in a controlled manner allowing the varying of only one independent variable at a time. The variables that were controlled included number of slaves, size of the contextual region, and size of a sub-problem. A sequential version of the algorithm was extracted from the parallel slave code for a benchmark.

The sequential and parallel versions were executed on the same machine to eliminate disparities due to machine capabilities and performance tuning.

The software includes statistical data collection in the code for determining the elapsed time for each execution of the program. In addition, the Unix 'time' command was utilized to gather the same statistics.

Numerous timings were obtained at off-peak operating times. Testing was normally conducted at night after determining that system usage was at a minimum. The first series of 10 timings were taken on an 8 -node Sequent Symmetry at the University of North Florida. A second series of 3 timings were taken on a 26-node Sequent Symmetry at Argonne National Laboratory in Chicago, IL.

The testing results have been compiled and are included in Appendix C. 


\section{Chapter 6}

\section{DISCUSSION OF RESULTS}

The graphic results from the AHE algorithm were quite impressive. Both the chest image (see Figures 14 and 15 on page 31) and the brain image (see Figures 16 and 17 on page 32) benefitted greatly from the processing. However, the purpose of this work is not to verify the results of the image processing, but to improve the time performance.

As mentioned in Chapter 5, three independent variables were tested. These were the number of slave processes, the size of the contextual region, and the size of the sub-problem. The brain image was used for all testing. However, it should be noted that the choice of the image does not affect the performance of the algorithm. The numerical results are included in Appendix C. A graphic representation of these results are shown in Figures 18 through 22 . Note that the sequential benchmark is indicated by the upright bar on the left of the graph, while the parallel timings are indicated by the lines on the chart. Each graph represents a single contextual region size. A separate line is included for each sub-problem size. The number of slaves utilized is indicated on the X-axis. The time in seconds is indicated on the Y-axis.

The results from the first set of timings, where the contextual region was set at $8 \times 8$, proved to be less than inviting. The amount of improvement hardly seemed worth the effort (see Figure 18 on page 33). The best case, for this contextual 


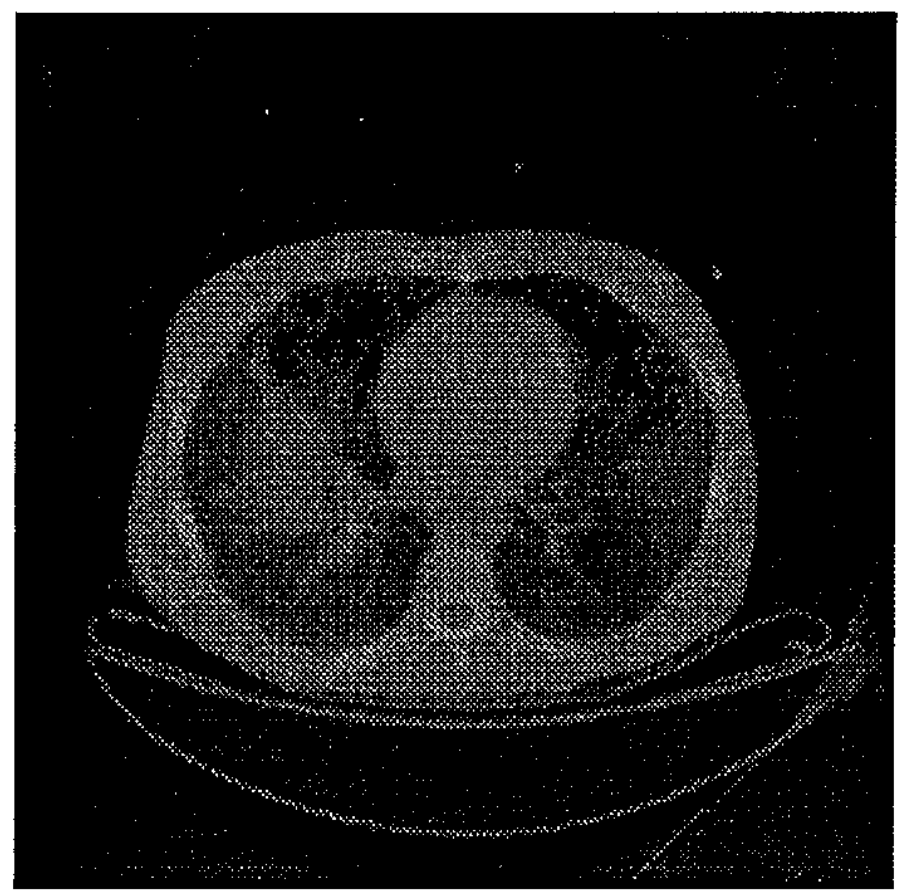

Figure 14: Chest - Original Image.

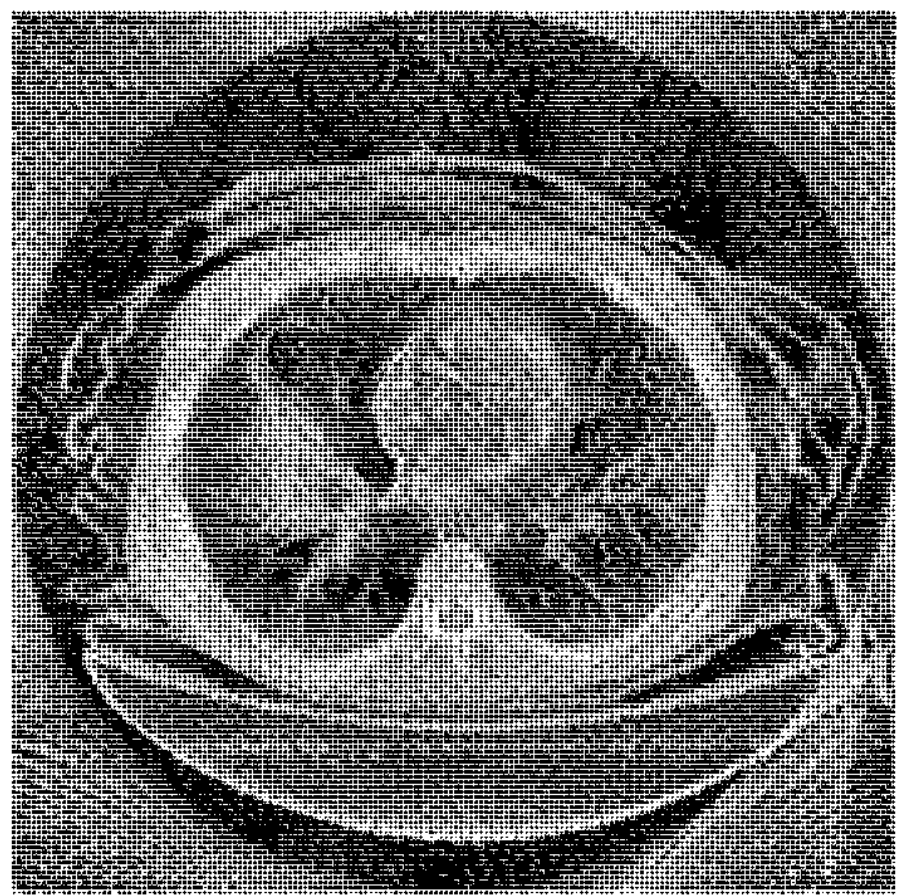

Figure 15: Chest - after AHE with contextual region of $64 \times 64$. 


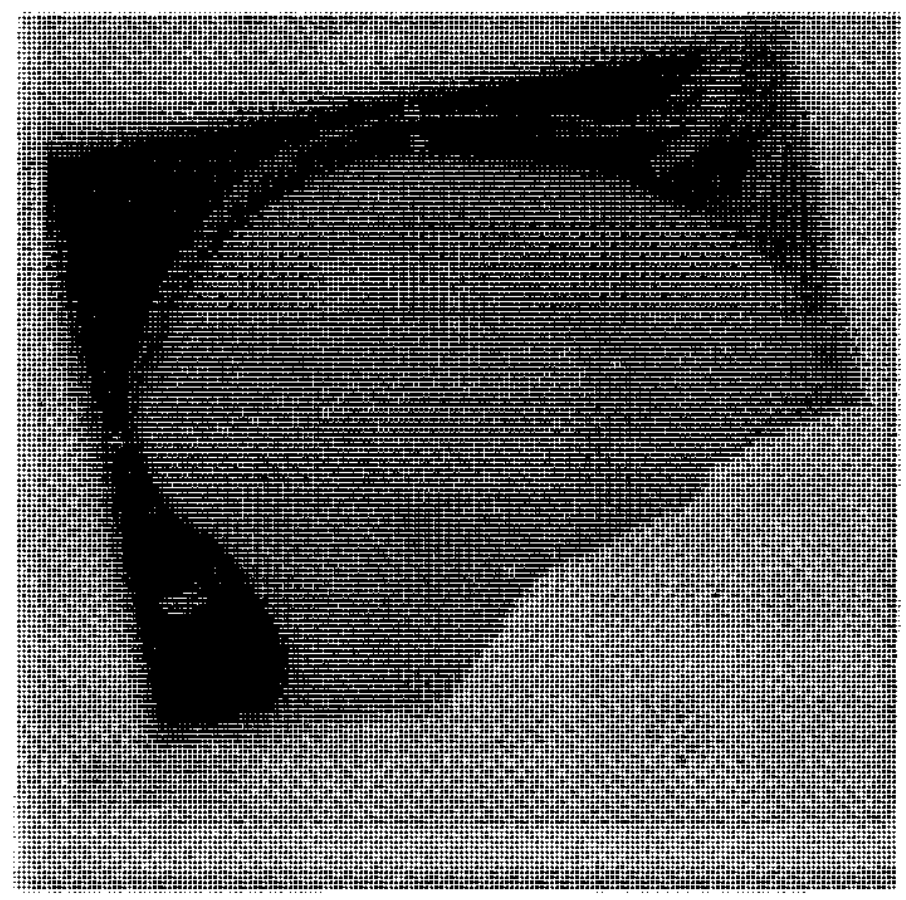

Figure 16: Brain - Original Image.

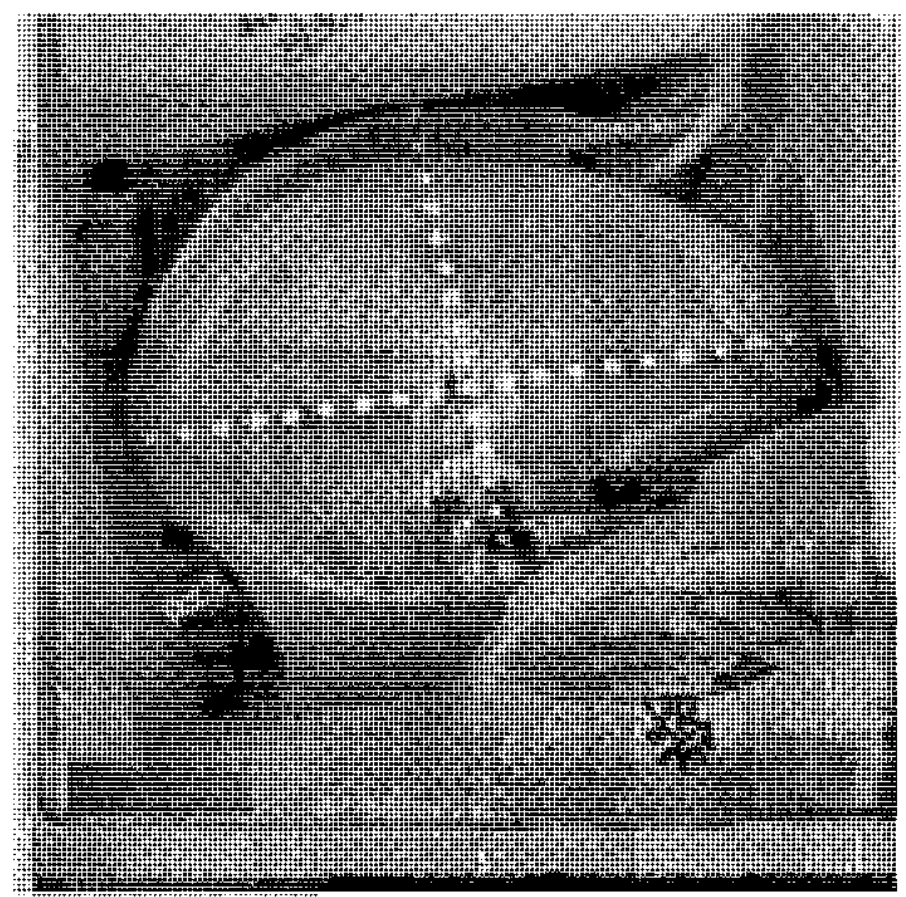

Figure 17: Brain - after AHE with contextual region of 64 x 64 . 


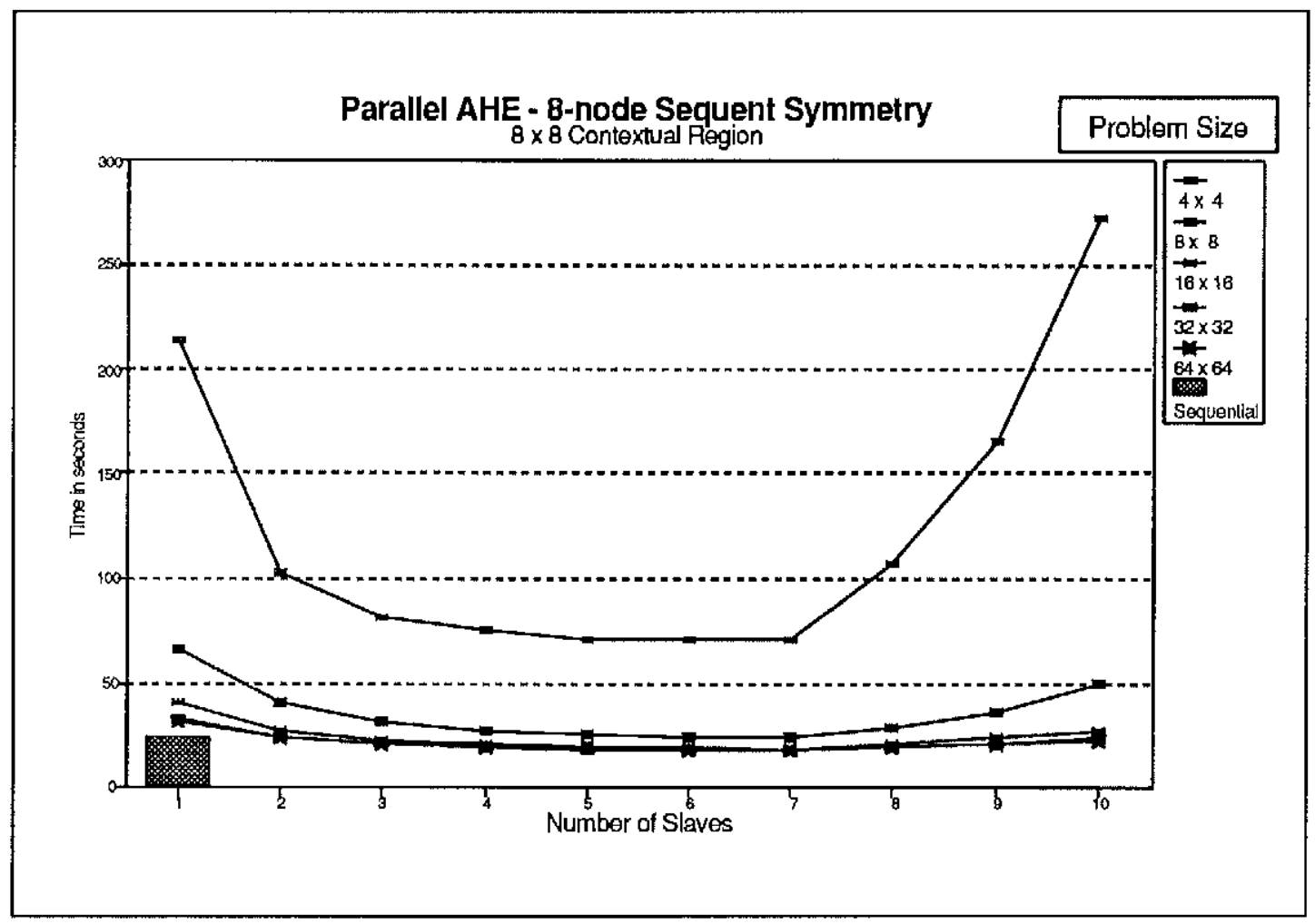

Figure 18: AHE Results - Contextual Region of $8 \times 8$.

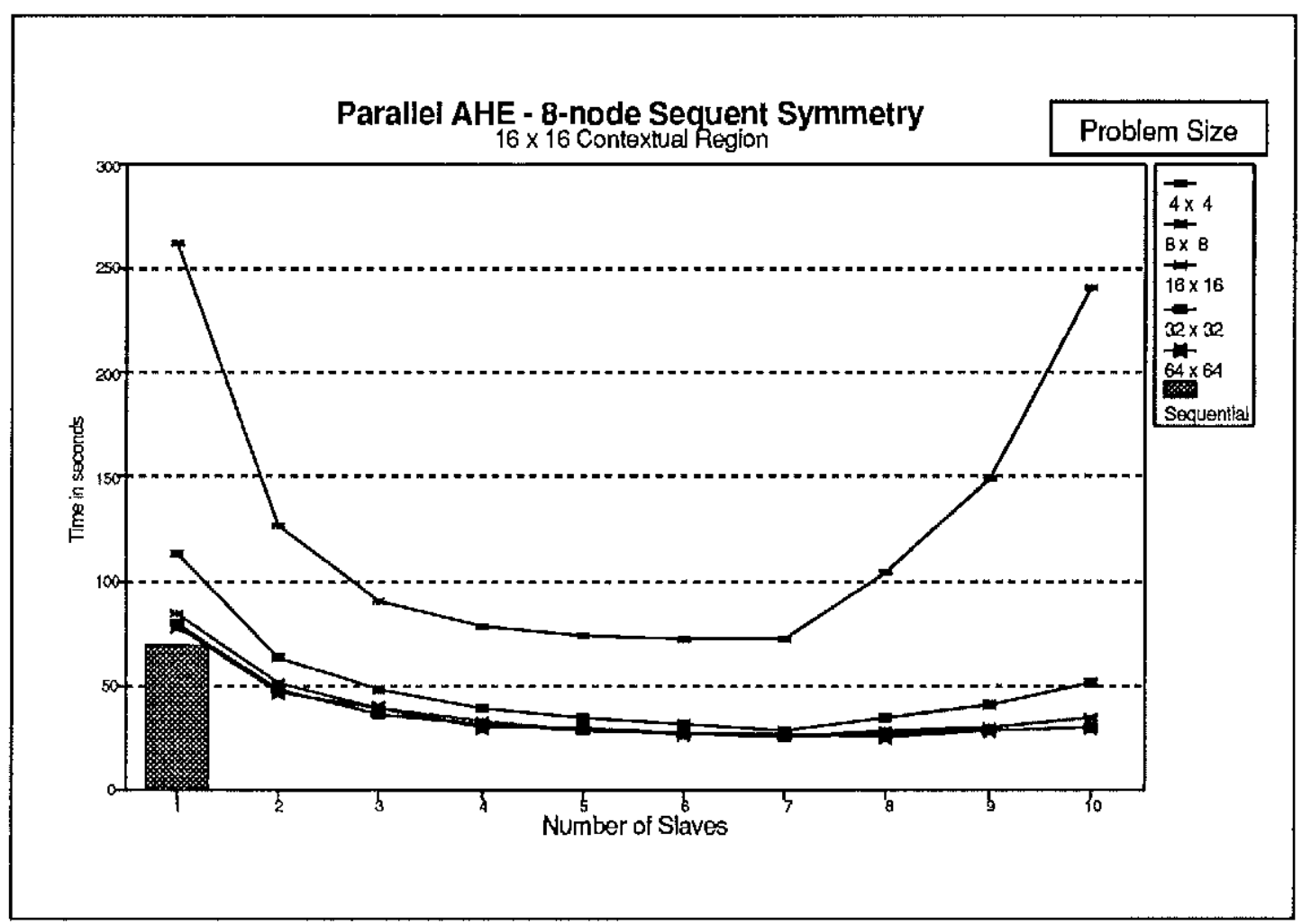

Figure 19: AHE Results - Contextual Region of 16 x 16 . 


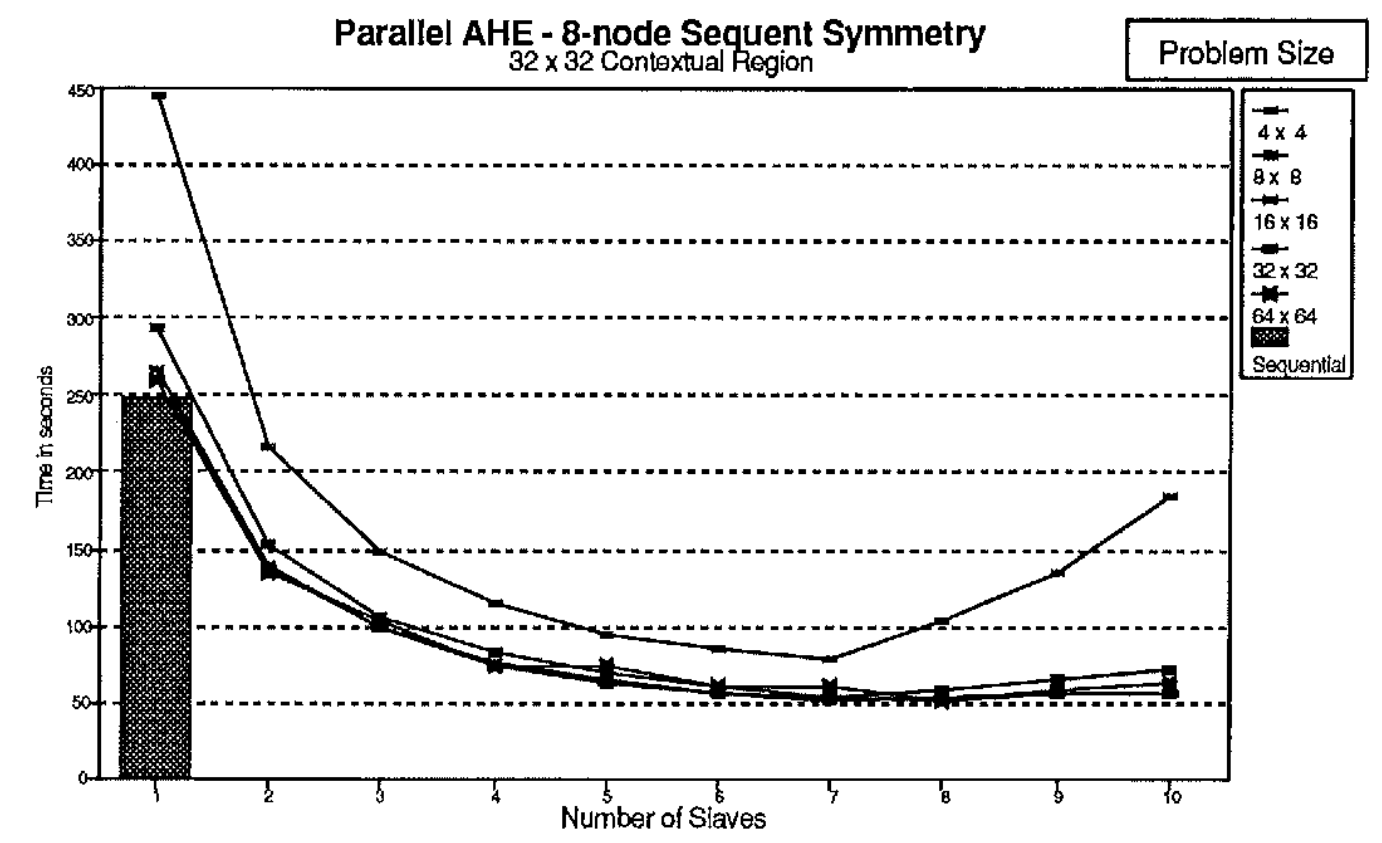

Figure 20: AHE Results - Contextual Region of $32 \times 32$.

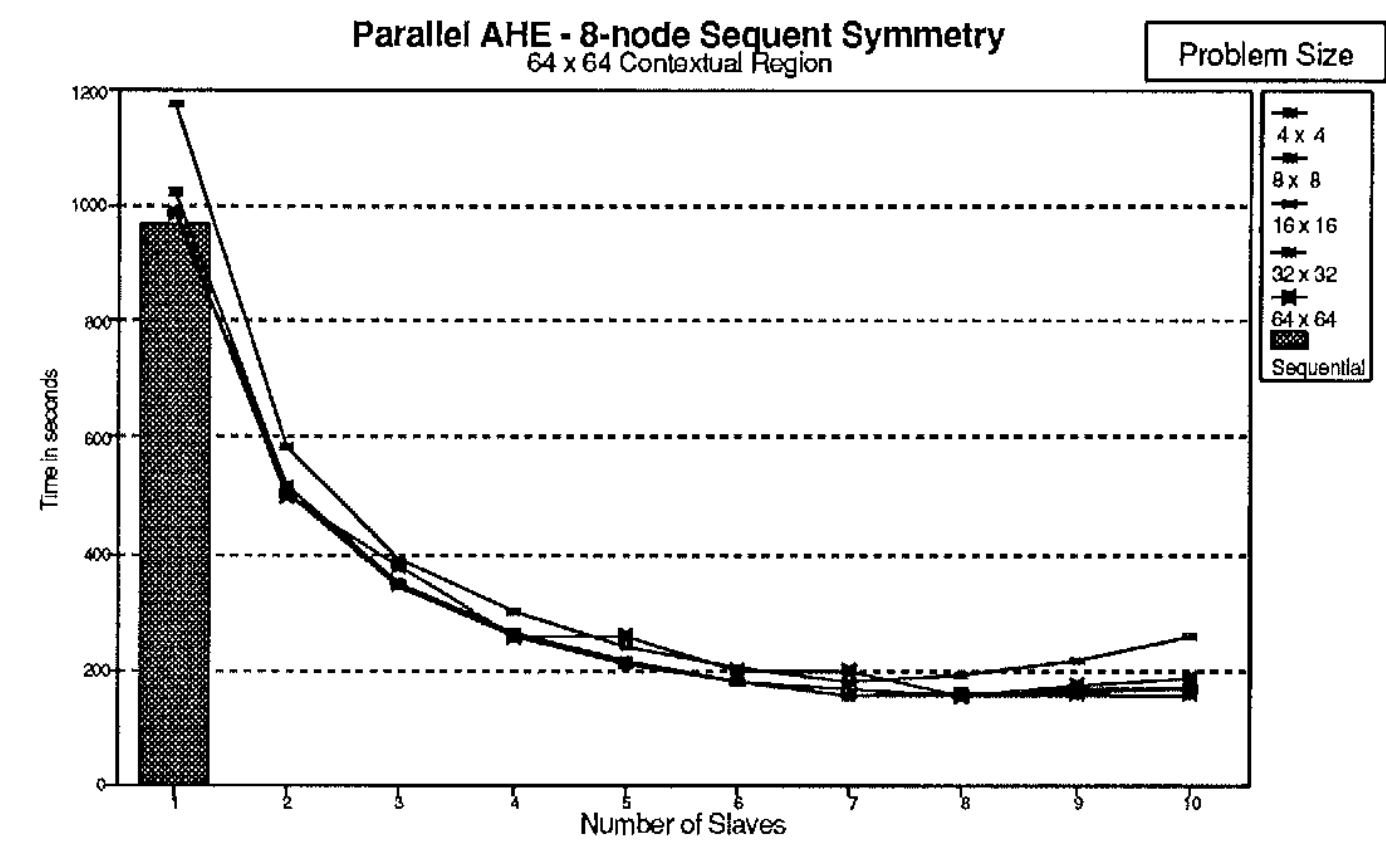

Figure 21: AHE Results - Contextual Region of $64 \times 64$. 
region size, was where the sub-problem size was $64 \times 64$. The sequential benchmark was 23.8 seconds, while the parallel version with seven CPUs (a master and six slaves) completed the identical task in 17.3 seconds.

It is also noted that for extremely small sub-problems sizes, the performance was over an order of magnitude greater in some cases than for larger sub-problem sizes (see Figure 18 on page 33). Both of the above performances are due to the effect of granularity with respect to parallelism. Simply stated, if the problem is too small, any speed-up contributed by the parallelism will be more than offset by the coordination overhead. As the size of the contextual region is increased, the effect of parallelism slowly begins to show. With a contextual region of $32 \times 32$, a more desirable comparison with the sequential benchmark is obtained (see Figure 20 on page 34$)$.

Increasing the contextual region size to $64 \times 64$ yields the best results with a speedup of approximately 6.5:1 (see Figure 21 on page 34). According to Mr. Cromartie of UNC-CH, the largest popular contextual region size is one-sixteenth of the image size. Thus, the contextual region variable was limited to $64 \times 64$.

An interesting phenomena occurred as the number of slaves was increased. At first the results appear to approximate the function $1 / \mathrm{n}$ where $\mathrm{n}$ is the number of slaves. Whereas perfect speed-up can not be realized due to communication and coordination overhead, the curve is close. However, as the number of slaves surpassed 7 on the first set of timings, the execution time increased. Note that this 
occurred at n-1 slaves where $n$ is the number of available processors in the machine.

The maximum benefit is realized when each process has its own processor. As the number of slaves increases beyond $\mathrm{n}-1$, there is more than one process sharing one or more of the processors. Multiple processes sharing a single processor increase overhead resulting in performance degradation.

The relationship between the number of processes and processors, however straightforward, seemed to beckon further support. With the assistance of Dr. Butler, the computing services at Argonne National Laboratory were obtained for additional testing. Due to the high amount of system usage at Argonne, the testing variables were limited. A contextual region of $64 \times 64$ was utilized, only varying the sub-problem size and the number of slaves. Also, testing for each possible slave count was bypassed in favor of sampling for the theorized trend. The resulting graph is included in Figure 22 on page 37.

It was estimated that the maximum performance would be at $n-1$ slaves where $n$ was 26 for the 26-node Sequent Symmetry at Argonne. With the very small subproblem size of $4 \times 4$, the speed-up reversed at 18 slaves. Evidently the overhead was too great.

For the remaining cases, the trend reverses at about 22 to 24 slaves. This is lower than expected. However, it was noted that system usage was not minimal during any of the testing periods. Thus, some of the slaves spent time waiting for 


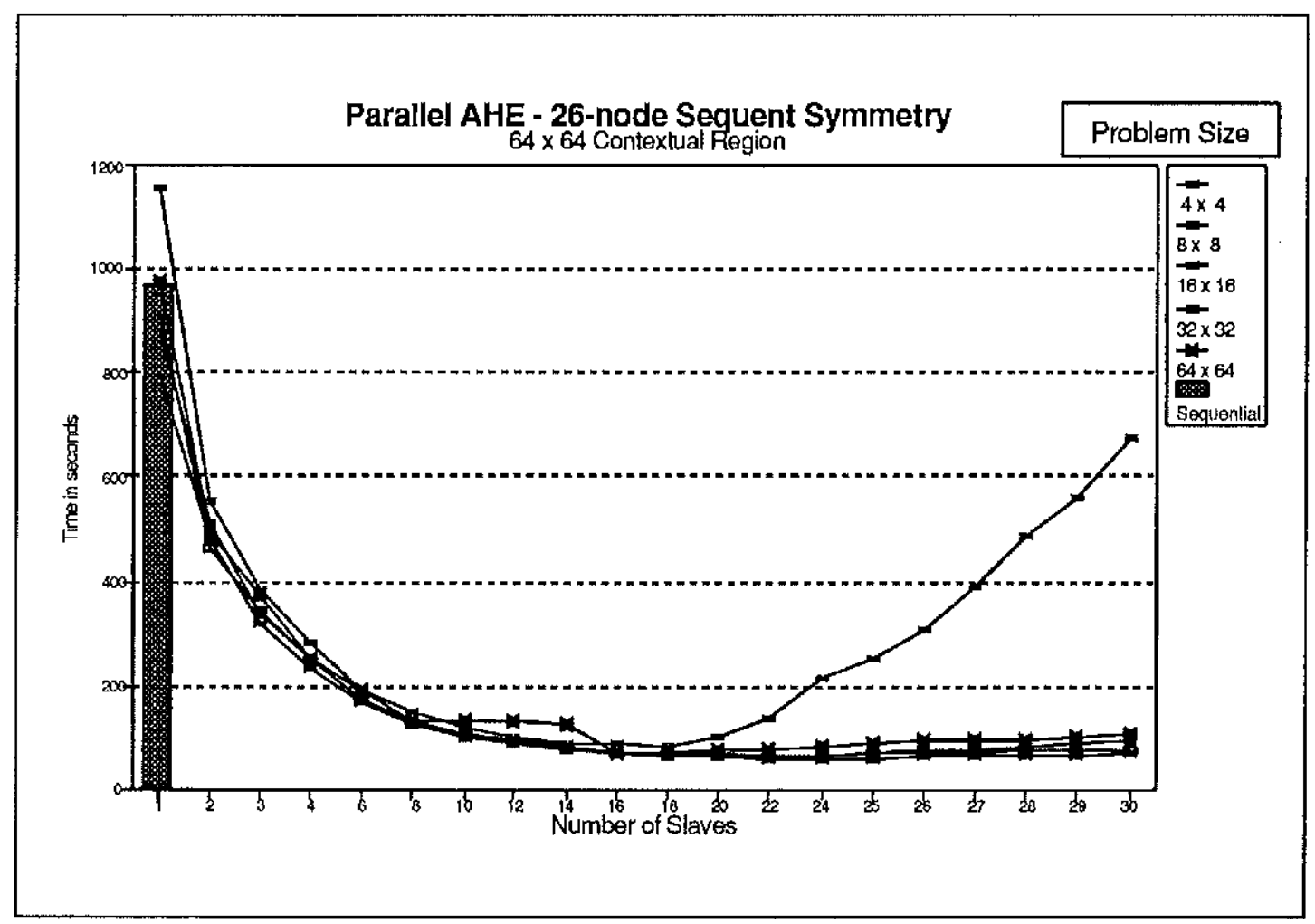

Figure 22: AHE Results - 26-node machine.

unrelated processes to relinquish processor control. This resulted in an overall increase in execution time.

Note however, that even though all 26 nodes were not available solely for the execution of this program, a worthwhile speedup was still realized. Where the 8-node machine yielded a best speed-up of approximately 6.5:1, the 26-node machine yielded a maximum speed-up of 16.5:1. 


\section{Chapter 7}

\section{CONCLUSIONS}

The hypothesis of this work is restated here:

There are parallel processing techniques which will substantially improve the time performance of Adaptive Histogram Equalization.

The implementation and testing conducted supports the hypothesis.

In addition, a method for handling border regions was developed and implemented.

Also, the application of a Look-Up Table served well to reduce the number of required computations. Whereas this probably did not affect the outcome of the testing, as both the sequential and parallel versions utilized this method, it did serve as an improvement to the overall implementation. 
Chapter 8

\section{FUTURE WORK}

There are numerous avenues that can be pursued to enhance this work. The research is by no means complete. One ultimate goal is to provide a real-time interactive system that would enable the user to manipulate the image as desired. Other goals are to enhance the algorithm to provide improved results. Several of these thoughts are included in the sections below. They are by no means a complete enumeration of the possibilities.

\subsection{SIMD architecture}

The parallel implementation of AHE in this thesis was performed on a Multiple Instruction Multiple Data (MIMD) machine. In this type of architecture, each processor can work on a problem independently or in coordination with other processors on the same machine. A different type of architecture is Single Instruction Multiple Data (SIMD). In this scenario each processor is executing the identical instruction in lock step on different data.

The SIMD machine requires a different design process during algorithm development and implementation. Different constraints are placed on the software. Current SIMD machines typically have less local memory per processing element than an MIMD machine. Accessing non-local memory is expensive. However, in 
spite of some of these limitations, there may still be major advantages for utilizing this type of hardware.

\subsection{Interactive viewing of image during processing}

Due to the length of time required to process an image, it may be desirable to display partial results as the job is in progress. The user could invoke a process that would first display the original image. The user could then specify a contextual region size for the AHE processing. As the image is processed, the partial solutions could be super-imposed over the original image. The user would have the option of interrupting the process by specifying a new contextual region size, or terminating the process, or allowing the process to complete normally.

The method of problem subdivision included in this implementation is to utilize a checkerboard type pattern. The original image is simply divided into small squares and handed out for processing. Clearly this would not be an acceptable visual display as portions of the image were completed. A different method that would be easy to implement would be to have each problem contain every nth pixel. Then, as sub-problems are solved, the effect would be a more gradual and smooth transition of the image. This method is depicted in Figure 23 on page 41. Here the original image is divided into 16 sub-problems. Each pixel is labeled by its sub-problem number. 
Dividing the image into interlaced sub-problems:

Each slave processes the set of pixels identified with a particular process number. As the results are returned and displayed, the effect is a gradual change vice a chcckerboard effect dcpicted by the boxes.

\begin{tabular}{|rrrr|rrrr|rrrr|rrrr|}
\hline 1 & 2 & 3 & 4 & 1 & 2 & 3 & 4 & 1 & 2 & 3 & 4 & 1 & 2 & 3 & 4 \\
5 & 6 & 7 & 8 & 5 & 6 & 7 & 8 & 5 & 6 & 7 & 8 & 5 & 6 & 7 & 8 \\
9 & 10 & 11 & 12 & 9 & 10 & 11 & 12 & 9 & 10 & 11 & 12 & 9 & 10 & 11 & 12 \\
13 & 14 & 15 & 16 & 13 & 14 & 15 & 16 & 13 & 14 & 15 & 16 & 13 & 14 & 15 & 16 \\
\hline 1 & 2 & 3 & 4 & 1 & 2 & 3 & 4 & 1 & 2 & 3 & 4 & 1 & 2 & 3 & 4 \\
5 & 6 & 7 & 8 & 5 & 6 & 7 & 8 & 5 & 6 & 7 & 8 & 5 & 6 & 7 & 8 \\
9 & 10 & 11 & 12 & 9 & 10 & 11 & 12 & 9 & 10 & 11 & 12 & 9 & 10 & 11 & 12 \\
13 & 14 & 15 & 16 & 13 & 14 & 15 & 16 & 13 & 14 & 15 & 16 & 13 & 14 & 15 & 16 \\
\hline 1 & 2 & 3 & 4 & 1 & 2 & 3 & 4 & 1 & 2 & 3 & 4 & 1 & 2 & 3 & 4 \\
5 & 6 & 7 & 8 & 5 & 6 & 7 & 8 & 5 & 6 & 7 & 3 & 5 & 6 & 7 & 8 \\
9 & 10 & 11 & 12 & 9 & 10 & 11 & 12 & 9 & 10 & 11 & 12 & 9 & 10 & 11 & 12 \\
13 & 14 & 15 & 16 & 13 & 14 & 15 & 16 & 13 & 14 & 15 & 16 & 13 & 14 & 15 & 16 \\
\hline 1 & 2 & 3 & 4 & 1 & 2 & 3 & 4 & 1 & 2 & 3 & 4 & 1 & 2 & 3 & 4 \\
5 & 6 & 7 & 8 & 5 & 6 & 7 & 8 & 5 & 6 & 7 & 8 & 5 & 6 & 7 & 8 \\
9 & 10 & 11 & 12 & 9 & 10 & 11 & 12 & 9 & 10 & 11 & 12 & 9 & 10 & 11 & 12 \\
13 & 14 & 15 & 16 & 13 & 14 & 15 & 16 & 13 & 14 & 15 & 16 & 13 & 14 & 15 & 16 \\
\hline
\end{tabular}

Figure 23: Dividing the image into interlaced sub-problems.

\subsection{The AHE Family of Algorithms}

Research at the University of North Carolina at Chapel Hill has produced other members of the AHE family of algorithms [Pizer87][Zimmerman89]. Among them are Weighted Adaptive Histogram Equalization (WAHE) and Clipped Adaptive Histogram Equalization (CLAHE). Current work by Mr. Cromartie is being conducted on Adaptive Histogram Equalization using Edge Detection. Certainly, each of these may benefit from the application of parallelism. This is a field that needs further exploration. 


\subsection{Three-dimensional AHE}

The advent of three-dimensional non-invasive imaging suggests the application of AHE in three dimensions. The concept of areas of low contrast in two dimensions can be expanded into the third dimension. As with two-dimensional AHE, this would be a pre-processing step prior to image display. The author has witnessed three-dimensional visualization tool development at $\mathrm{UNC}-\mathrm{CH}$ and believes that 3-D AHE may be of benefit. 


\section{REFERENCES}

\section{[Austin87]}

J.D. Austin, Stephen M. Pizer, "A Multiprocessor Adaptive Histogram Equalization Machine," The University of North Carolina at Chapel Hill, (1987).

[Boyle87]

J. Boyle, R. Butler, T. Disz, B. Glickfield, E. Lusk, R. Overbeek, J.

Patterson, R. Stevens, Portable Programs for Parallel Processors, Holt, Rinehart and Winston, Inc., (1987).

[Butler90]

R. Butler, Unpublished technical memo, (1990).

[Ericksen90]

J.P. Ericksen, S.M. Pizer, J.D. Austin, "MAHEM: A Multiprocessor Engine for Fast Contrast-Limited Adaptive Histogram Equalization," Medical Imaging IV, SPIE Proceedings, 1233, (1990).

[Hummel75]

R.A. Hummel, "Histogram Modification Techniques," Computer Graphics and Image Processing, 4, (1975), pp. 209-224.

[Hummel77]

R.A. Hummel, "Image Enhancement by Histogram Transformation,"

Computer Graphics and Image Processing, 6, (1977), pp. 184-195.

[Ketcham76]

D.J. Ketcham, "Real-Time Image Enhancement Techniques," Seminar on Image Processing, Pacific Grove California, Hughes Aircraft Company, 1976, p. $1-6$.

[Pizer81a]

S.M. Pizer, "An Automatic Intensity Mapping for the Display of CT Scans and Other Images", Medical Image Processing: Proceedings of the VIIth International Meeting on Information Processing in Medical Imaging, Stanford, California, Stanford University, (1981), pp. 276-309.

[Pizer81b]

S.M. Pizer, "Intensity Mappings for the Display of Medical Images", Functional Mapping of Organ Systems: 11th Annual Symposium on the Sharing of Computer Programs and Technology in Nuclear Medicine, New Orleans, Louisiana, P.D. Esser (ed.), Society of Nuclear Medicine, (1981), pp. 205-218. 
[Pizer86]

S.M. Pizer, "Adaptive Histogram Equalization for Automatic Contrast Enhancement of Medical Images", Application of Optical Instrumentation in Medicine, XIV: Medical Imaging, Processing, and Display and Picture Archiving, and Communication Systems (PACS IV) for Medical Applications, (1986), pp. 242-250.

[Pizer87]

S.M. Pizer, "Adaptive Histogram Equalization and Its Variations", Computer Vision, Graphics, and Image Processing, 39, (1987), pp. 355-368.

[Zimmerman89]

J.B. Zimmerman, S.B. Cousins, M.E. Frisse, K.M. Hartzell, M.G. Kahn, "A Psychophysical Comparison of Two Methods for Adaptive Histogram Equalization," Washington University, St. Louis, Missouri 63130. 


\section{APPENDIX A}

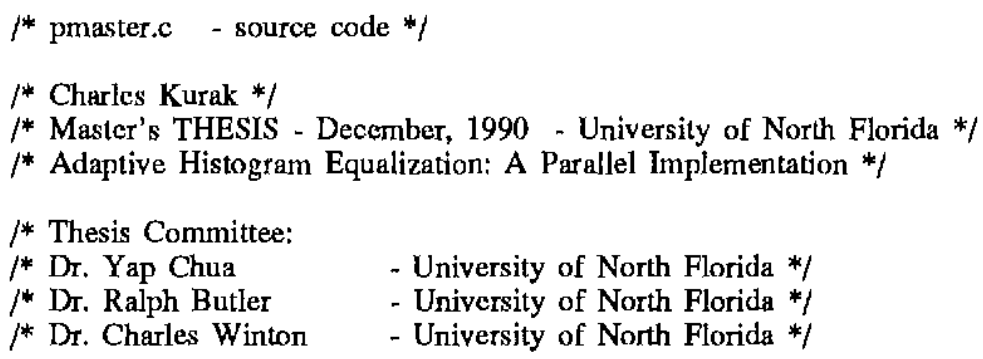

/* slave message types */ \#define REQUEST_WORK 3 


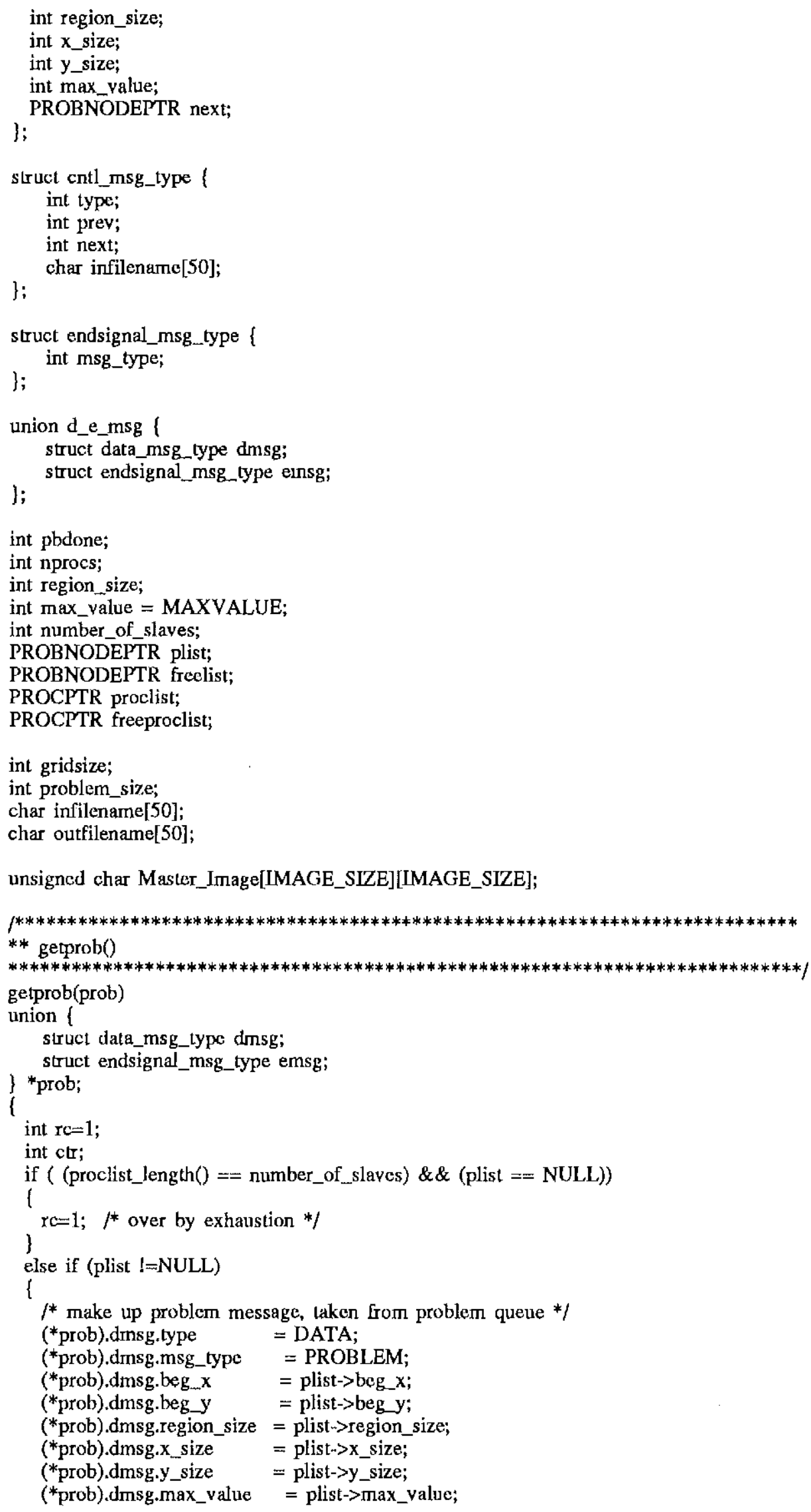




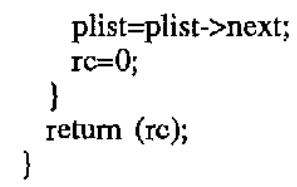




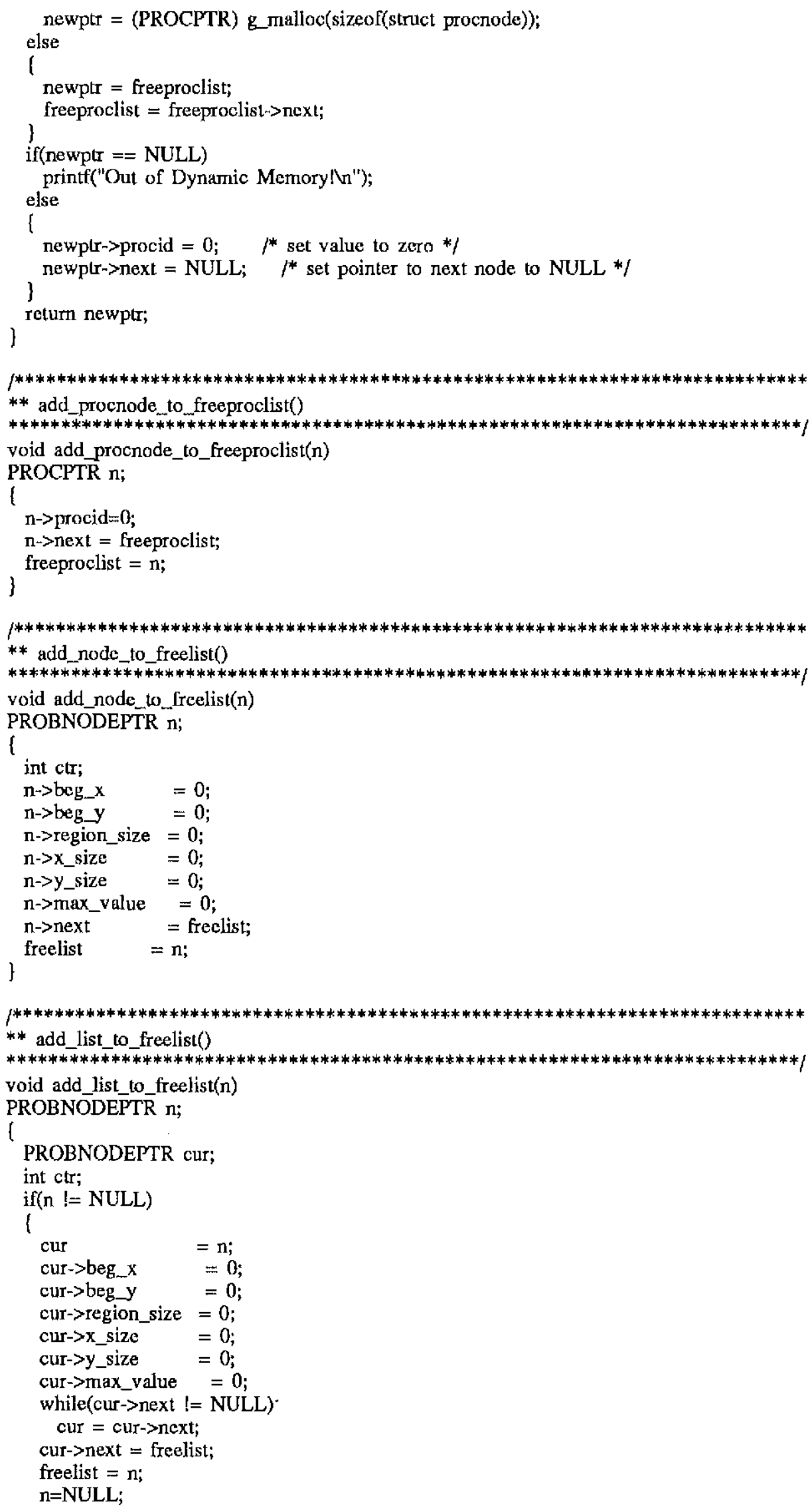




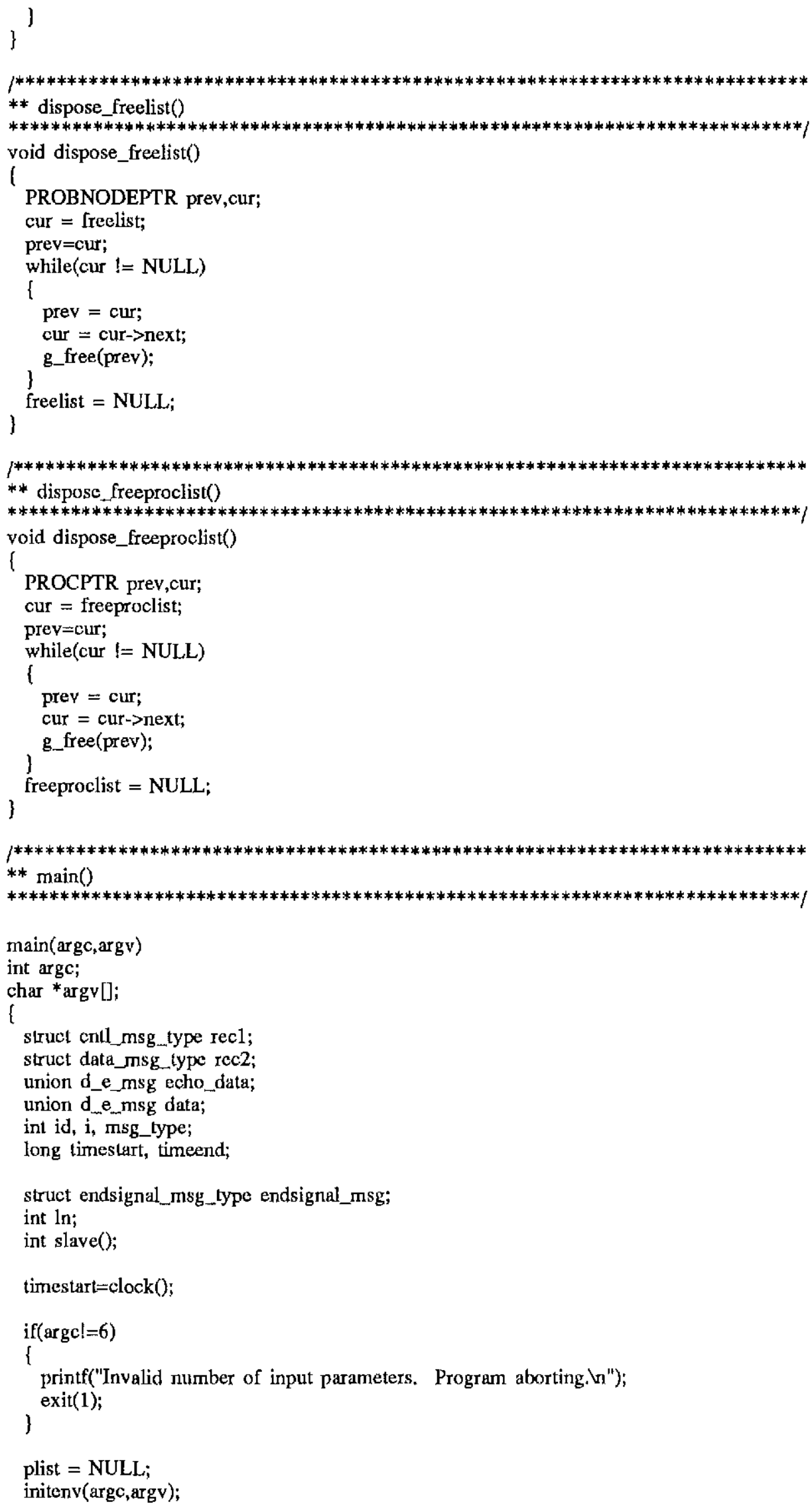


create_procgroup $($;

number_of of slaves=get_nslaves();

$/^{*}$ read in the contextual region size */

region_size=atoi $(\operatorname{argv}[1])$;

$f^{*}$ read in the problem_size */

problem_size=atoi $(\operatorname{argv}[2])$;

$1^{*}$ read in the max_value *1

max_value=atoi(argv[3]);

$f^{*}$ read in the input filename */

strepy(infilename, argv[4]);

$/$ * read in the output filename */

strcpy(outfilename, argv[5]);

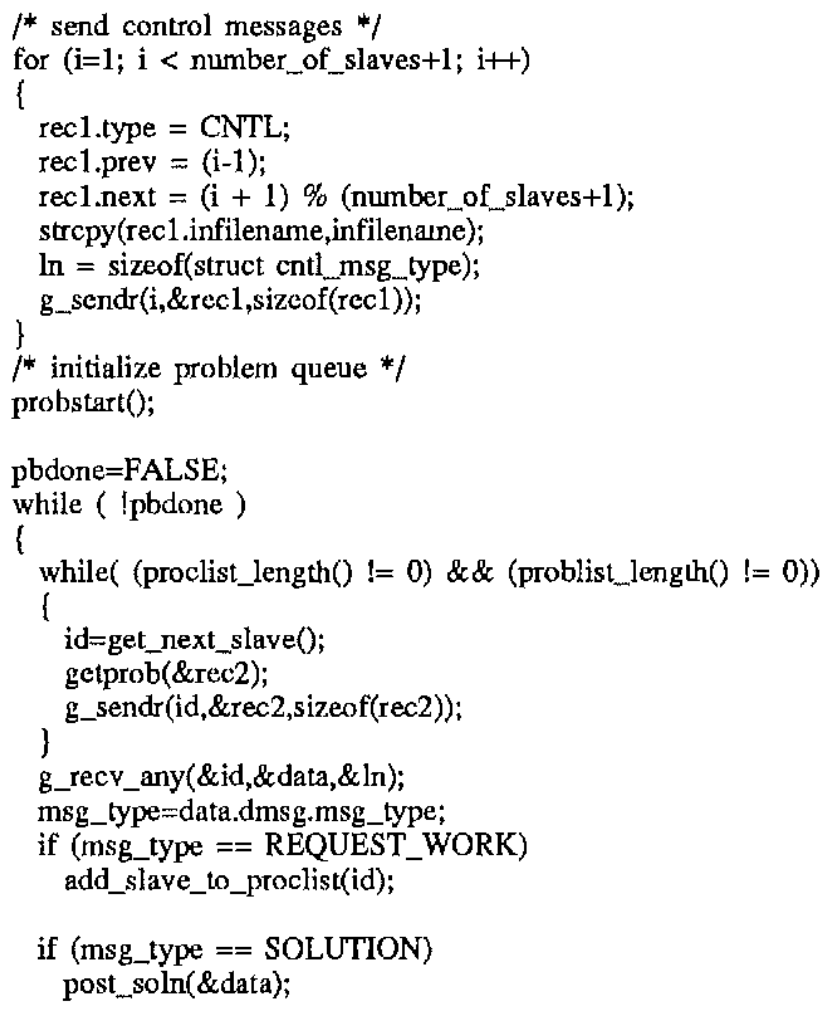

$/^{*}$ All the slaves are waiting for work \&\& there is no more work */ if $($ proclist_length ()$==$ number_of_slaves) \& \& (problist_length $(0==0)$ ) 


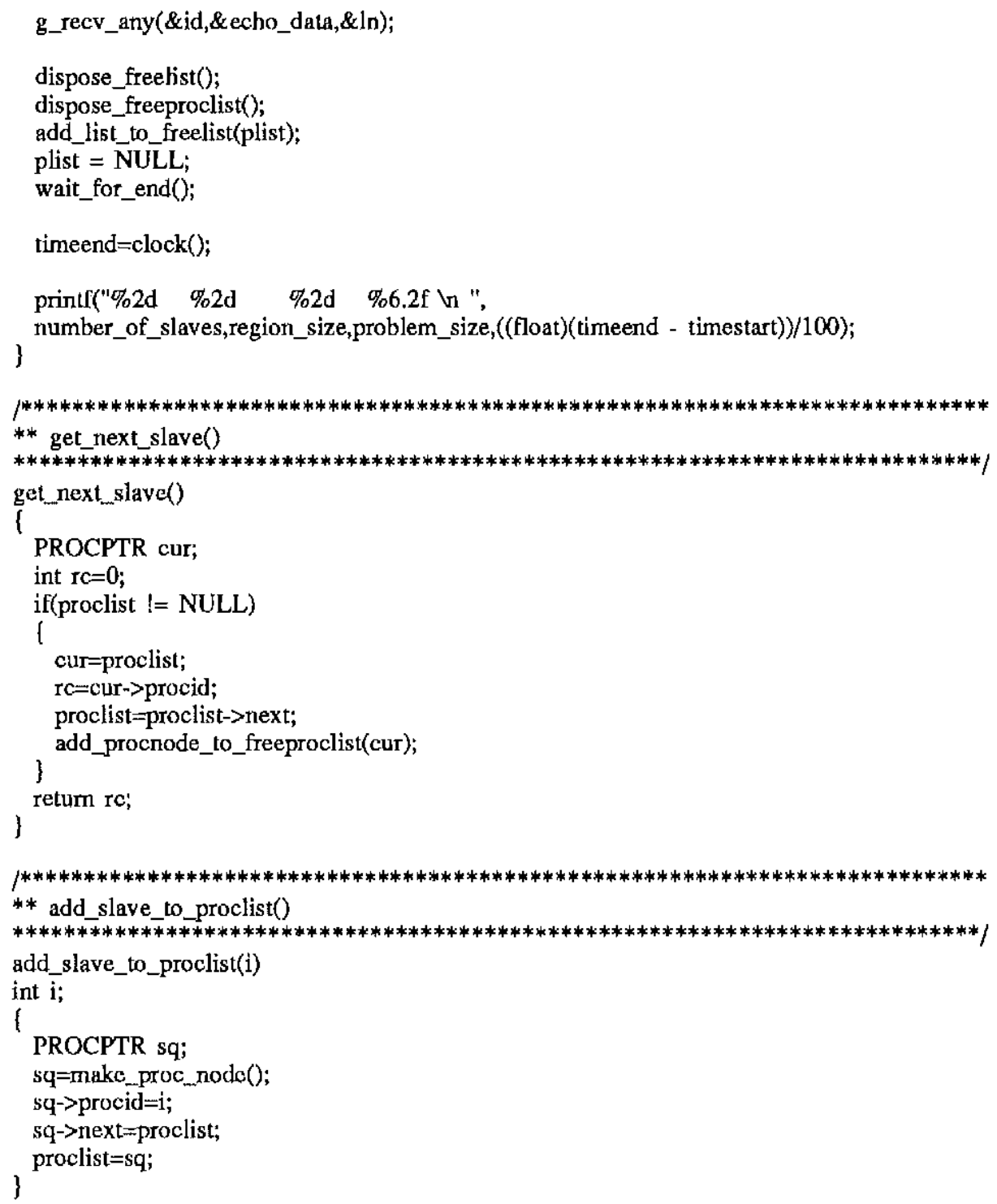




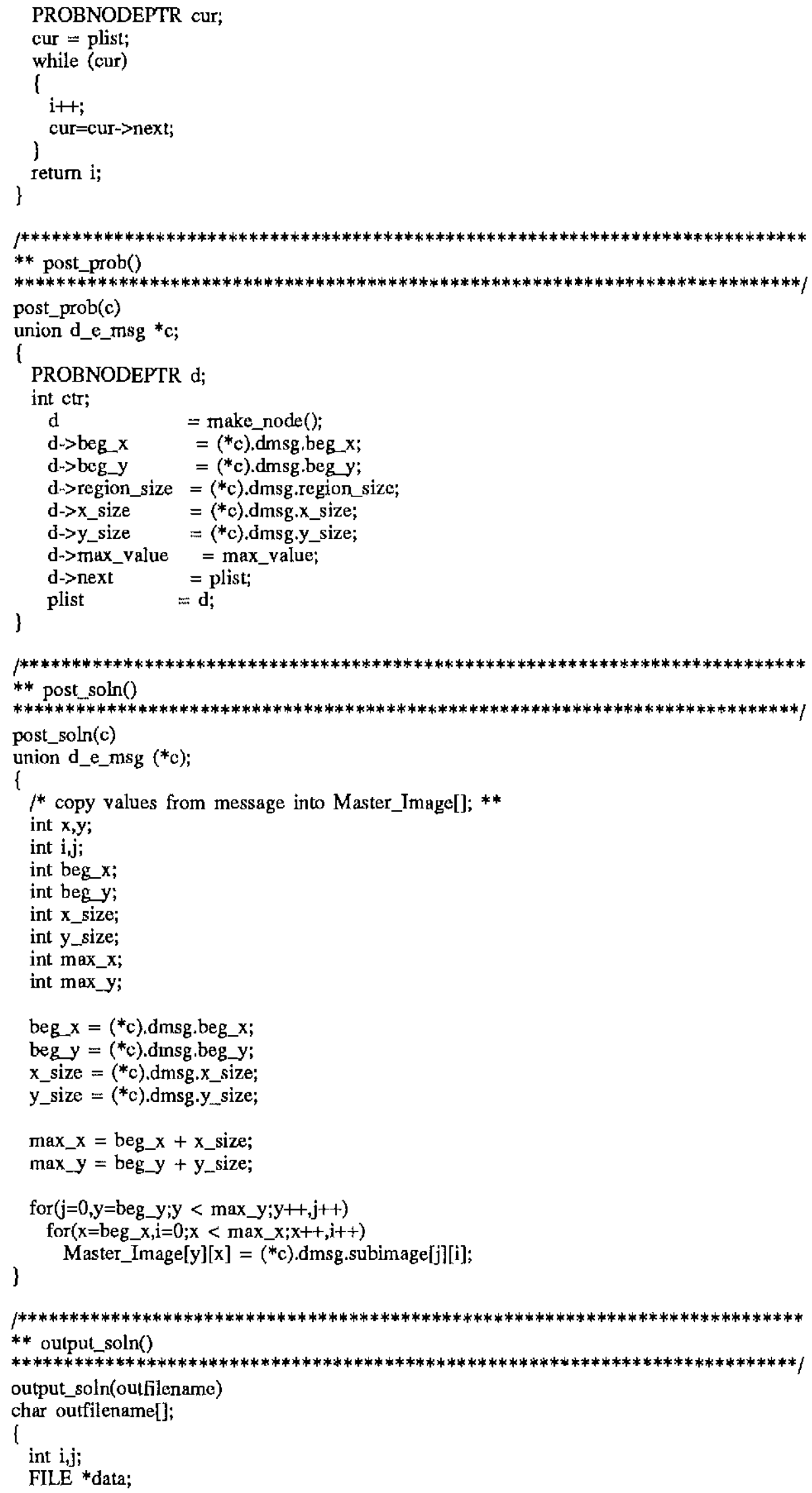




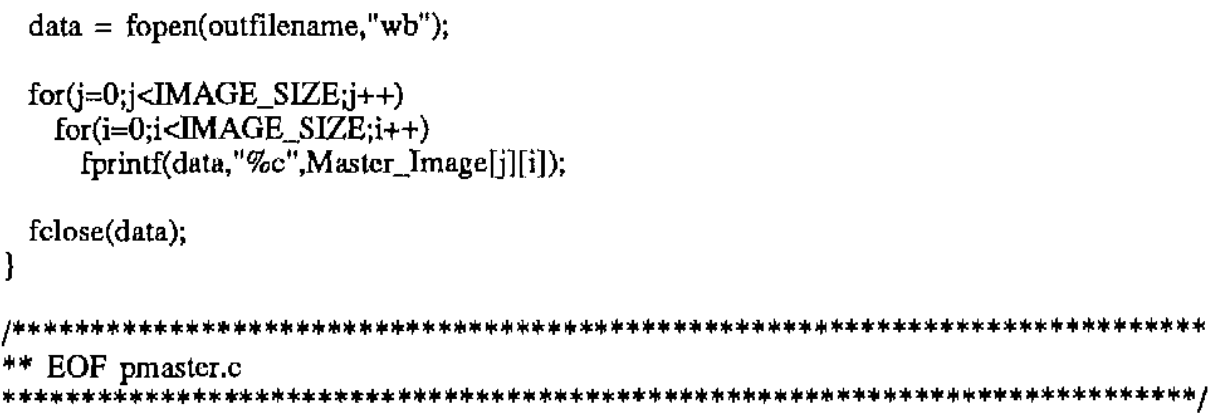




\section{APPENDIX B}

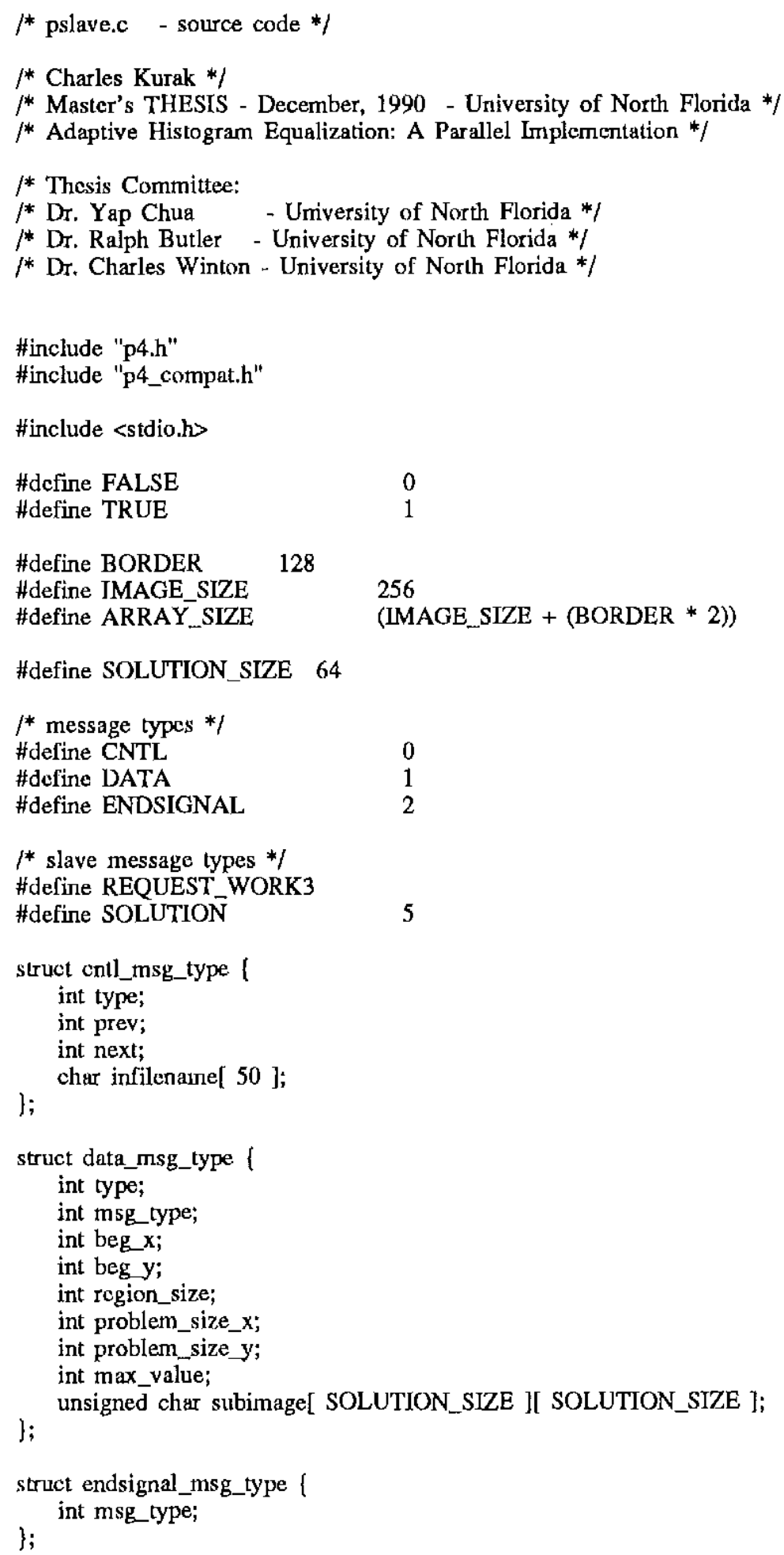




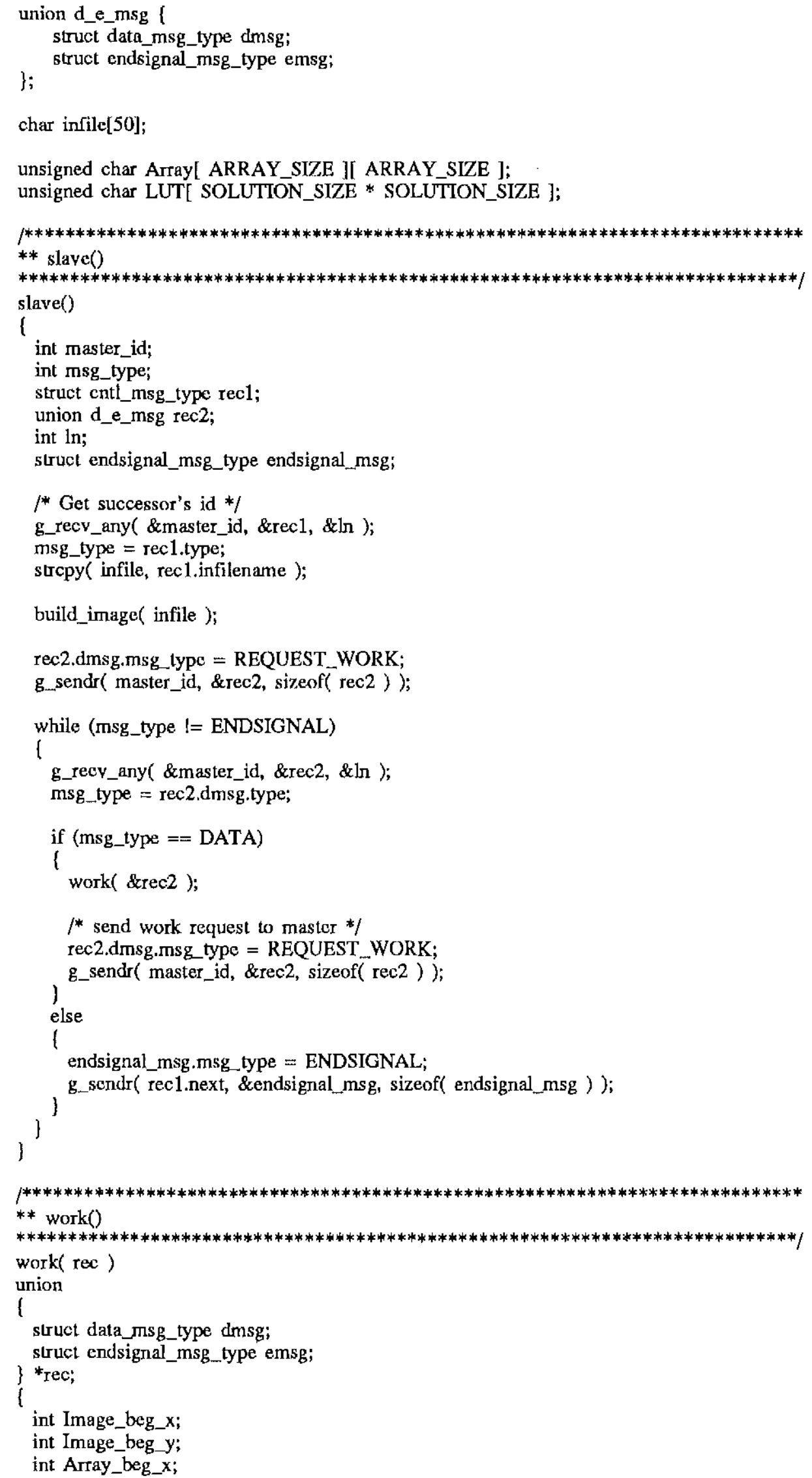




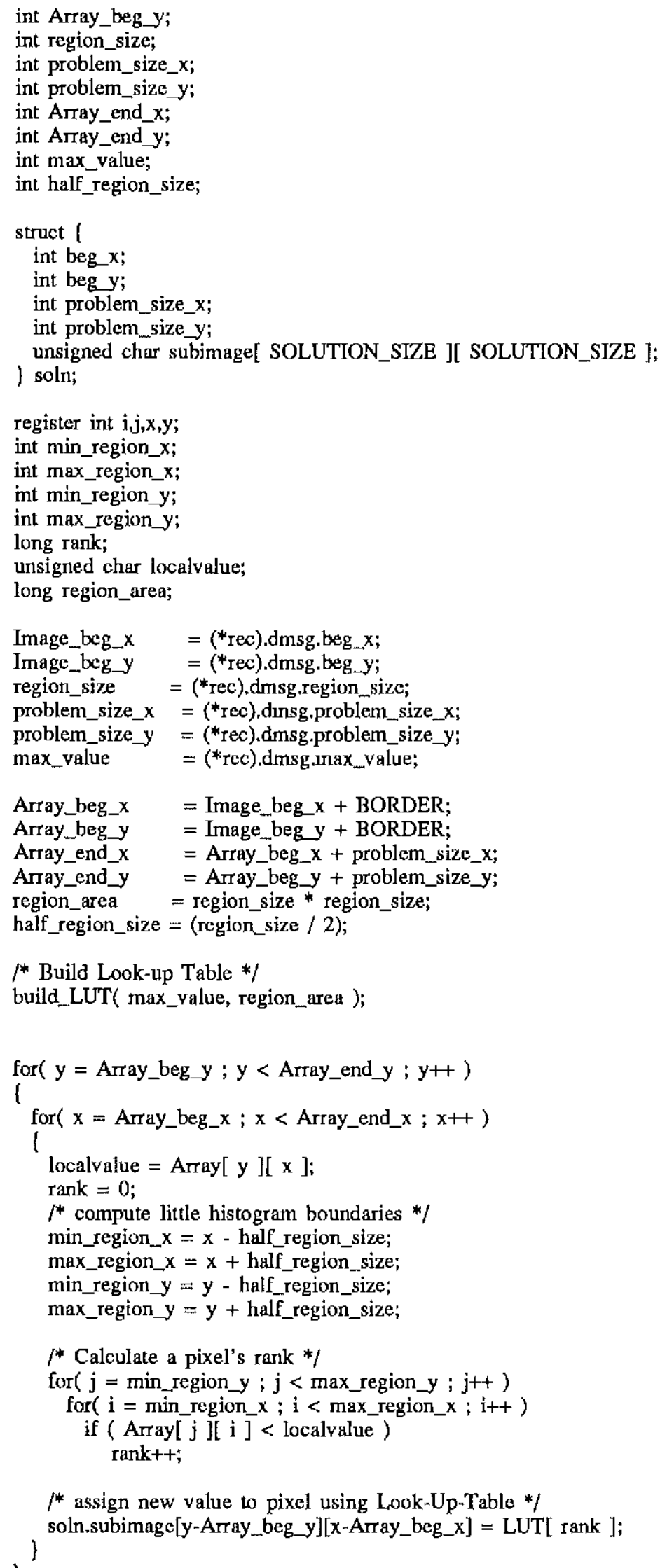




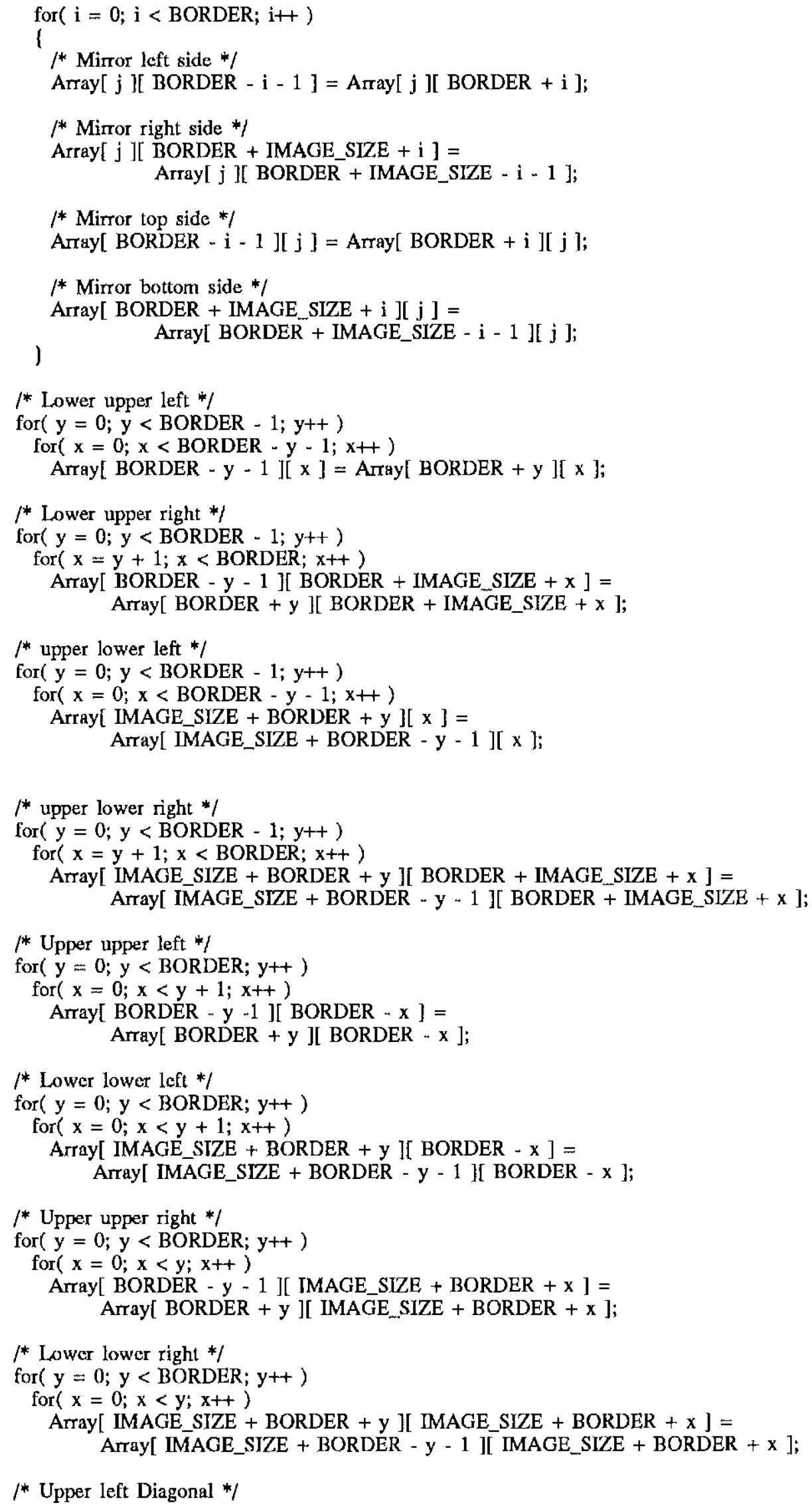




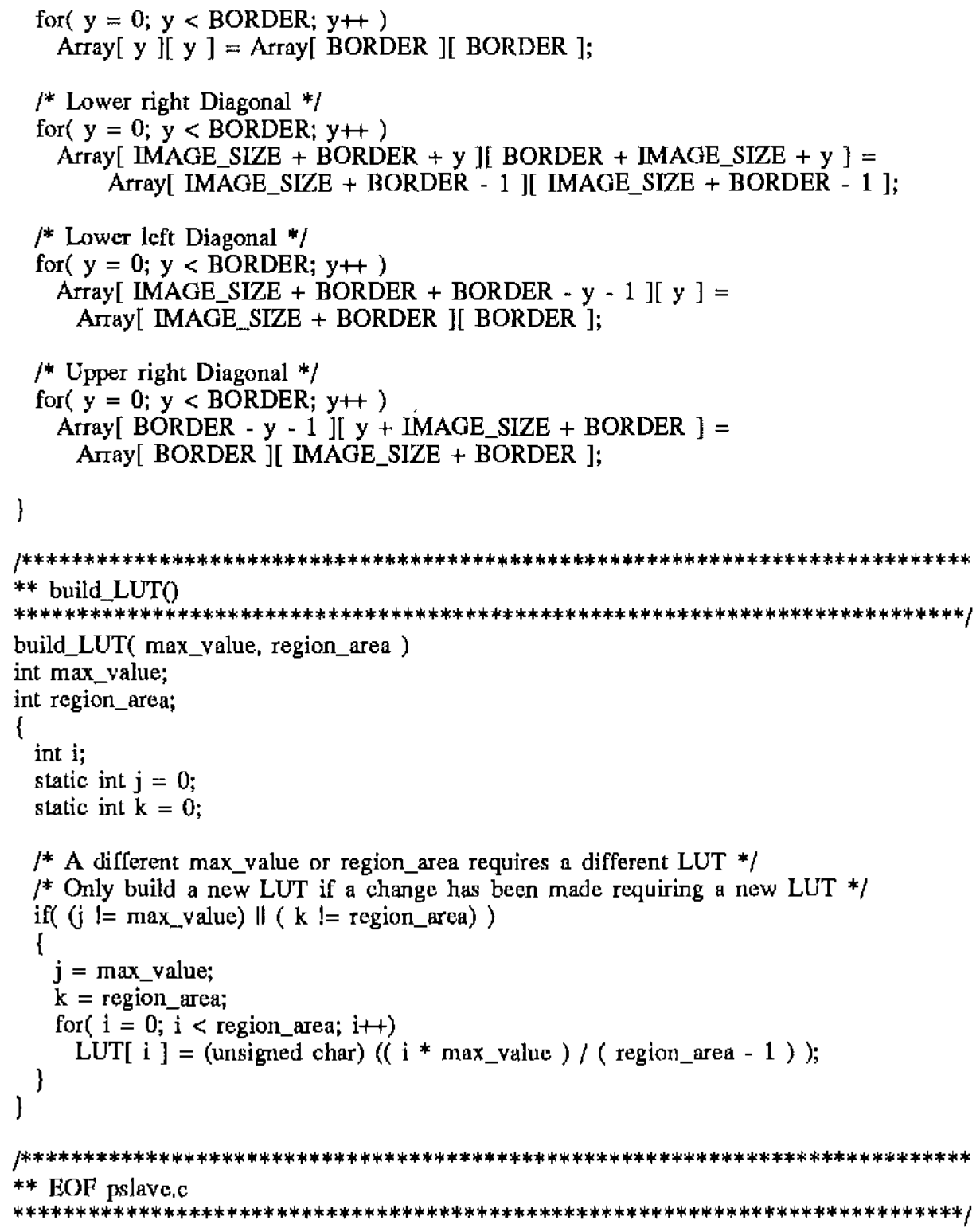


APPENDLX C

Test Results: Contextual Region of $8 \times 8$.

Hardware: UNF Sequent Symmetry

(8-node machine)

\begin{tabular}{rrrrrrr} 
& $\begin{array}{c}\text { Number } \\
\text { of } \\
\text { Sequential }\end{array}$ & $\underline{5}$ & \multicolumn{4}{c}{ Parallel Problem Size } \\
23.8 & 1 & 214.5 & 66.2 & 39.5 & 33.0 & 31.7 \\
& 2 & 102.2 & 39.8 & 26.5 & 23.6 & 22.9 \\
& 3 & 80.5 & 31.2 & 22.5 & 20.6 & 20.2 \\
& 4 & 74.7 & 27.3 & 20.5 & 18.6 & 18.7 \\
& 5 & 70.9 & 24.8 & 18.9 & 18.1 & 18.6 \\
6 & 71.0 & 23.8 & 18.5 & 17.7 & 17.3 \\
7 & 70.9 & 23.4 & 17.9 & 17.4 & 17.5 \\
& 8 & 106.9 & 28.7 & 20.3 & 19.0 & 18.7 \\
9 & 165.7 & 35.4 & 23.4 & 21.0 & 20.2 \\
10 & 272.4 & 49.5 & 26.6 & 22.9 & 22.3
\end{tabular}

All times in seconds.

Table 1: 8-node Sequent Symmetry, 8 × 8 Contextual Region.

Test Results: Contextual Region of $16 \times 16$.

Hardware: UNF Sequent Symmetry

(8-node machine)

\begin{tabular}{|c|c|c|c|c|c|c|}
\hline \multicolumn{3}{|c|}{ Number } & \multicolumn{4}{|c|}{ Parallel Problem Sizc } \\
\hline Sequential & Slaves & $\underline{4 \times 4}$ & $\underline{8 \times 8}$ & $\underline{16 \times 16}$ & $\underline{32 \times 32}$ & $\underline{64 \times 64}$ \\
\hline 69.9 & 1 & 262.3 & 112.2 & 84.9 & 79.0 & 77.5 \\
\hline & 2 & 126.7 & 62.3 & 50.6 & 47.4 & 46.9 \\
\hline & 3 & 89.8 & 47.3 & 39.1 & 36.3 & 38.1 \\
\hline & 4 & 77.8 & 38.5 & 32.1 & 30.6 & 30.2 \\
\hline & 5 & 73.4 & 33.8 & 28.8 & 27.9 & 30.3 \\
\hline & 6 & 71.4 & 30.7 & 26.5 & 26.0 & 26.4 \\
\hline & 7 & 71.4 & 28.6 & 24.9 & 24.4 & 26.6 \\
\hline & 8 & 103.3 & 33.7 & 27.4 & 26.1 & 25.0 \\
\hline & 9 & 149.3 & 39.5 & 30.1 & 27.8 & 27.6 \\
\hline & 10 & 240.9 & 50.7 & 34.2 & 29.3 & 30.3 \\
\hline
\end{tabular}

All times in seconds.

Table 2: 8-node Sequent Symmetry, $16 \times 16$ Contextual Region. 
Test Results: Contextual Region of $32 \times 32$.

Hardware: UNF Sequent Symmetry (8-node machine)

\begin{tabular}{|c|c|c|c|c|c|c|}
\hline \multicolumn{3}{|c|}{ Number } & \multicolumn{4}{|c|}{ Parallel Problem Size } \\
\hline Sequential & Slaves & $\underline{4 \times 4}$ & $\underline{8 \times 8}$ & $16 \times 16$ & $32 \times 32$ & $\underline{64 \times 64}$ \\
\hline 251,0 & 1 & 445.0 & 293.9 & 266.8 & 260.5 & 259.4 \\
\hline & 2 & 217.2 & 153.6 & 139.6 & 136.8 & 135.5 \\
\hline & 3 & 149.2 & 106.4 & 97.9 & 97.9 & 104.5 \\
\hline & 4 & 115.1 & 83.0 & 76.4 & 74.8 & 74.4 \\
\hline & 5 & 95.5 & 69.1 & 64.2 & 63.3 & 74.6 \\
\hline & 6 & 85.2 & 59.8 & 55.7 & 55.6 & 60.6 \\
\hline & 7 & 79.6 & 54.4 & 51.2 & 52.7 & 60.0 \\
\hline & 8 & 103.0 & 58.2 & 52.9 & 52.3 & 51.6 \\
\hline & 9 & 135.7 & 64,0 & 54.8 & 55.9 & 57.1 \\
\hline & 10 & 184.5 & 71.0 & 56.8 & 56.7 & 62.3 \\
\hline
\end{tabular}

All times in seconds.

Table 3: 8-node Sequent Symmetry, 32 x 32 Contextual Region.

Test Results: Contextual Region of $64 \times 64$

Hardware: UNF Sequent Symmetry (8-node machine)

\begin{tabular}{rrrrrrr} 
& $\begin{array}{c}\text { Number } \\
\text { of } \\
\text { Sequential }\end{array}$ & \multicolumn{5}{c}{ Paraltel Problem Size } \\
\cline { 5 - 6 } 974.5 & $\underline{\text { Slaves }}$ & $\underline{4 \times 4}$ & $\underline{8 \times 8}$ & $\underline{16 \times 16}$ & $\underline{32 \times 32}$ & $\underline{64 \times 64}$ \\
& 1 & 1174.3 & 1024.7 & 997.2 & 989.1 & 987.5 \\
& 2 & 583.7 & 518.1 & 505.3 & 502.3 & 500.4 \\
& 3 & 394.1 & 349.3 & 341.5 & 347.3 & 376.6 \\
& 4 & 298.4 & 265.5 & 259.0 & 257.2 & 256.6 \\
5 & 241.7 & 214.7 & 211.9 & 210.5 & 255.0 \\
6 & 203.4 & 180.9 & 178.3 & 180.1 & 194.9 \\
7 & 177.0 & 157.4 & 154.8 & 164.8 & 195.3 \\
8 & 193.1 & 159.6 & 155.4 & 155.8 & 154.9 \\
9 & 217.7 & 162.1 & 156.7 & 166.1 & 173.0 \\
& 10 & 256.6 & 166.0 & 157.4 & 165.2 & 187.8
\end{tabular}

All times in seconds.

Table 4: 8-node Sequent Symmetry, 64 x 64 Contextual Region. 
Test Results: Contextual Region: $64 \times 64$.

Hardware: Argonne National Laboratory Sequent Symmetry (26-node machine)

$\begin{array}{rrrrrrr} & \begin{array}{c}\text { Number } \\ \text { of } \\ \text { Sequential } \\ \text { Slaves }\end{array} & \underline{4 \times 4} & \underline{8 \times 8} & \underline{16 \times 16} & \underline{32 \times 32} & \underline{64 \times 64} \\ 974.5 & 1 & 1159.8 & 882.7 & 794.3 & 915.4 & 979.2 \\ & 2 & 556.0 & 512.5 & 481.4 & 462.2 & 494.8 \\ & 3 & 386.5 & 335.7 & 318.1 & 341.2 & 371.1 \\ & 4 & 280.6 & 253.9 & 233.6 & 250.5 & 250.6 \\ 6 & 190.1 & 172.8 & 165.0 & 173.9 & 189.4 \\ 8 & 147.5 & 126.9 & 126.0 & 128.9 & 128.6 \\ 10 & 118.4 & 101.2 & 102.9 & 105.1 & 128.3 \\ 12 & 99.7 & 88.1 & 86.3 & 92.0 & 127.9 \\ & 14 & 89.5 & 77.8 & 76.7 & 83.5 & 121.8 \\ 16 & 85.8 & 70.6 & 70.6 & 71.2 & 72.4 \\ 18 & 83.0 & 66.5 & 66.4 & 71.3 & 72.2 \\ 20 & 99.4 & 63.0 & 62.4 & 71.9 & 75.1 \\ 22 & 134.4 & 61.6 & 60.0 & 60.8 & 77.7 \\ 24 & 217.0 & 64.9 & 58.8 & 63.5 & 82.0 \\ 25 & 249.4 & 67.5 & 59.8 & 68.3 & 87.0 \\ 26 & 306.1 & 73.5 & 61.4 & 69.7 & 91.5 \\ 27 & 389.5 & 73.8 & 62.8 & 71.2 & 92.4 \\ 28 & 488.1 & 82.4 & 62.3 & 73.6 & 97.1 \\ 29 & 561.2 & 87.1 & 65.4 & 76.8 & 98.3 \\ & & 672.2 & 93.7 & 69.8 & 75.7 & 103.6\end{array}$

All times in seconds.

Table 5: 26-node Sequent Symmetry. 


\section{VITA}

Charles William Kurak Jr. has a Bachelor of Arts degree from Thomas A. Edison State College of New Jersey in Natural Sciences and Mathematics 1984 and expects to receive a Master of Science in Computer and Information Sciences from the University of North Florida, May 1991. Dr. Yap S. Chua of the University of North Florida is serving as Charles' thesis adviser. Charles has been employed as a computer programmer analyst by the United States Navy since 1984, and has been a paid Graduate Research Assistant at UNF from May, 1990 to December, 1990.

Charles' extensive professional and academic programming experience includes use of C, Pascal, and BASIC in the Unix, MS-DOS, and combined LAN environments. He has developed utilities for professional systems use including graphics, file viewing, communications, and database systems. His academic work encompasses 2-D and 3-D imaging and graphics, ray tracing, communications, systems software, data modeling, human-machine interfacing, and multi-processing.

Charles is listed in Who's Who Among Students in American Colleges and Universities, 1990. He has been a member of the IEEE and the IEEE Computer Society and the Association for Computing Machinery (ACM) since 1989. He has been serving as the Chair of the Student Chapter of the ACM at UNF (ACM-UNF) since 1989. Charles has presented his research work to the Jacksonville FL Region of the Mathematical Association of America and ACM-UNF. Charles has been married for the past twelve and one-half years. He has one son, age 11 years. 